\title{
Corrélations investissement-épargne et mobilité internationale des capitaux*
}

\author{
Jean-Pierre Berdot** \\ Université de Poitiers \\ Gérard Kébabdjian*** \\ Université de Paris VIII \\ Jacques Léonard ${ }^{* * * *}$ \\ Université de Poitiers
}

\section{Introduction}

L'hypothèse de parfaite mobilité des capitaux est sous-jacente à presque toutes les contributions récentes en macroéconomie internationale. Cette hypothèse se développe sur l'intuition selon laquelle la mobilité du capital serait devenue très forte dans le monde contemporain à la suite de la libéralisation et de l'intégration financières intervenues durant les vingt dernières années. L'idée qu'il se serait produit un vaste mouvement de « globalisation financière " est à la base des analyses habituelles sur l'instabilité financière internationale et la propagation internationale des crises financières. De façon surprenante, toutefois, la plupart des travaux empiriques ne parviennent pas à valider cette hypothèse.

$\mathrm{Au}$ contraire, dans leur article inaugural, Feldstein et Horioka (1980) (ci-après notés $\mathrm{FH}$ ) ont soutenu que, même entre les principaux pays industrialisés, la mobilité du capital pouvait être sévèrement limitée (voir aussi

* Les auteurs remercient leurs trois rapporteurs anonymes, et tout particulièrement le dernier d'entre eux, pour leurs précieux commentaires el remarques constructives. L'entière responsabilité de cet article leur incombe néanmoins. Version définitive : avril 2001.

** Professeur à l'Université de Poitiers, membre du Centre de Recherche sur l'Intégration Économique et Financière (CRIEF), Faculté de Sciences Économiques, 93 avenue du Recteur Pineau, 86022 Poitiers Cedex, e-mail : jean-pierre.berdot@univ-poitiers.fr

*** Protesseur à l'Université de Paris VIII, membre du Centre d'Études en Macroéconomie et Finance Internationale (CEMAFI), Université de Nice - Sofia Antipolis, 7 avenue Robert Schuman, 06050 Nice Cedex, e-mail : gkebabdjian@ univ-paris8.fr

**** Professeur à l'Université de Poitiers, membre du Centre de Recherche sur l'Intégration Économique et Financière (CRIEF), Faculté de Sciences Économiques, 93 avenue du Recteur Pineau, 86022 Poitiers Cedex, e-mail : jacques.leonard@univ-poitiers.Ir 
Feldstein (1983)) . Leur approche, qui s'appuie sur la corrélation investissement-épargne, a été ensuite largement acceptée et utilisée comme test du degré de mobilité internationale du capital. Une autre approche, également employée, examine dans quelle mesure la parité des taux d'intérêt (couverte ou non) est réalisée pour un pays ou pour un groupe de pays. L'approche par les taux d'intérêt fait clairement apparaître un degré élevé de mobilité (voir les recensions de Frankel (1992) ou d'Obsfeldt (1995)), ce qui semble contredire la première approche. Or, celles-ci sont complémentaires : la première correspond à un test macroéconomique et la seconde à un test microéconomique. Cet article s'intéresse au paradoxe soulevé par la première approche.

Vingt ans se sont écoulés depuis la publication de l'article de FH. En vingt ans, un nombre considérable de recherches ont été conduites pour tenter de réconcilier leurs résultats avec les indications fournies par l'approche microéconomique. Avec des variables supplémentaires ou des spécifications légèrement différentes, ces recherches empiriques ont repris la même problématique fondamentale; toutes ont confirmé, à des nuances près, le résultat de base de $\mathrm{FH}$, à savoir l'absence de tests significatifs du point de vue macroéconomique validant l'hypothèse d'une mobilité élevée du capital pour la période récente.

Les données accumulées suggèrent au contraire que la corrélation entre épargne et investissement est une régularité empirique robuste. Ces résultats jettent un doute considérable sur l'idée que les marchés nationaux de capitaux sont fortement interconnectés. Les corrélations positives entre les niveaux et les variations des taux d'épargne et d'investissement (établies pour les pays industrialisés et, dans une moindre mesure, pour les pays en développement, y compris sur les périodes les plus récentes) ont résisté à une grande variété d'objections économétriques.

Dans le débat d'idées, ce résultat négatif est même quelquefois utilisé pour soutenir que la «globalisation financière " n'est qu'une illusion sans fondement statistique. Concernant l'analyse économique, le résultat pose toutefois plus de problèmes qu'il n'apporte de réponses. Les interrogations ont donné naissance à une variété extraordinaire de travaux pour tenter de résoudre ce qu'il est convenu d'appeler aujourd'hui le «puzzle FeldsteinHorioka ».

On peut énoncer l'énigme de la façon suivante : si l'indicateur retenu par FH est une bonne mesure de la mobilité du capital, alors, selon la lecture habituelle des tests, l'hypothèse de mobilité devrait être rejetée. Mais rien n'assure que cette lecture soit la bonne ni que cet indicateur soit le seul pertinent. Le programme de recherche consiste alors à découvrir la pièce manquante du puzzle et à établir ce que mesurent exactement les « tests à la $\mathrm{FH}$ ».

Compte tenu de la prolifération et de la progressive complexité des travaux accumulés depuis vingt ans, il semble indispensable de reprendre les études économétriques de façon systématique. En actualisant et en analy- 
sant plus finement les estimations économétriques habituelles, des résultats neufs apparaissent. Ils donnent une image complètement différente de celle qui ressort de la littérature.

Notre étude établit deux résultats principaux. En premier lieu, même en nous limitant aux indicateurs habituels, et contrairement aux résultats considérés comme acquis, nos tests confortent l'idée d'un accroissement de la mobilité internationale des capitaux. En second lieu, un examen plus approfondi des relations entre épargne et investissement conduit à mettre en évidence une seconde série de preuves établissant, sur le plan macroéconomique, que la mobilité internationale des capitaux s'est effectivement accrue depuis le début des années quatre-vingt. Ces deux types de résultats permettent de réconcilier l'intuition empirique avec l'analyse économique et dissipent l'espèce de schizophrénie qui guette le macroéconomiste s'intéressant aux mouvements internationaux de capitaux.

Le plan de l'article est le suivant : la première section est une recension visant à expliciter les termes du puzzle tels qu'ils se posent aujourd'hui après vingt ans de controverses; la deuxième section reprend les termes standards pour effectuer des estimations économétriques actualisées et affinées et justifie, contrairement aux résultats habituels, l'idée d'une tendance à la globalisation financière établie sur la base des tests à la $\mathrm{FH}$ dans leur version simple; la troisième section intègre, dans la problématique habituelle à la $\mathrm{FH}$, des données complémentaires qui confortent l'existence d'une tendance à la globalisation financière.

\section{Le puzzle Feldstein-Horioka, vingt ans de mésaventures}

\section{$2.1 \quad$ Le point de départ}

Dans leur article de 1980, FH partaient d'une idée apparemment raisonnable: avec une mobilité du capital parfaite, «il ne devrait plus y avoir de relation entre l'épargne domestique et l'investissement domestique puisque l'épargne dans chaque pays doit réagir aux opportunités d'investissement à l'échelle mondiale alors que l'investissement dans chaque pays se trouve financé par le pool formé par le capital mondial » (p. 317). Pour tester la relation entre l'épargne et l'investissement et pour en apprécier le degré de corrélation (et non, bien évidemment, pour estimer une fonction d'investissement), les auteurs évaluaient la droite d'ajustement :

$$
i=\alpha+\beta s
$$

où $i$ et $s$ représentent les taux d'investissement et d'épargne (rapports entre investissement ou épargne intérieur(e) brut(e) et produit intérieur brut). 
Dans une économie fermée, ou pour une économie avec parfaite immobilité des capitaux, $\beta$ est égal à 1 par définition. Mais cela ne signifie pas - notons-le dès à présent car ce point est à la base des débats - que, sous les conditions de parfaite mobilité, $\beta$ doive nécessairement prendre la valeur 0 ni même que la parfaite mobilité des capitaux soit incompatible avec des valeurs de $\beta$ proches de 1 .

C'est toutefois sur ces conjectures que repose l'interprétation des premiers résultats des tests à la FH. Ultérieurement, Feldstein (1983) propose un modèle permettant de les soutenir. Il y explore la distinction usuelle entre les estimations en données chronologiques et les estimations en coupes transversales (alors que l'analyse de 1980 concernait uniquement les secondes). Aucun changement important n'apparaît au plan des résultats statistiques : l'hypothèse d'une corrélation épargne-investissement ne peut être rejetée, ce qui conforte la conclusion principale de $\mathrm{FH}$ : le marché international des capitaux est loin d'être aussi « globalisé » qu'on le croit. Pour autant, le résultat obtenu n'est nullement clair, d'autant que le modèle fait intervenir les niveaux d'investissement et d'épargne alors que les estimations portent sur les taux et que le modèle est très restrictif. La faiblesse de la modélisation théorique $^{1}$ a d'ailleurs été soulignée très tôt (voir le commentaire de Tobin (1983)).

Le plus inacceptable est de supposer, contre toute logique, que le $\beta$ résultant du modèle proposé (en séries temporelles) est le même que le $\beta$ défini dans une approche en coupes transversales parce que les deux $\beta$ mesurent en réalité des choses très différentes. Il est tout à fait possible d'imaginer des configurations dans lesquelles le $\beta$ en séries temporelles, $\beta_{T}$, est élevé alors que le $\beta$ en coupes, soit $\beta_{C}$, est faible, ou vice-versa. La confusion entre les deux coefficients est à l'origine de beaucoup d'idées reçues. Même quand la confusion est dénoncée (i.e. Obsfeldt (1995)), l'interprétation n'est pas toujours satisfaisante. Ainsi, l'idée que $\beta_{T}$ est une mesure de «court terme » tandis que $\beta_{C}$ est une mesure « de long terme », comme l'affirme Obsfeldt, repose sur une simple analogie fondée sur les différences classiques entre estimations de court terme et de long terme.

Même sous les simplifications du modèle de Feldstein, l'interprétation des résultats économétriques n'est pas évidente. Avec une parfaite mobilité du capital, par exemple, $\beta$ ne tend pas vers zéro. Le sens de variation de $\beta$ sous l'influence de l'augmentation de la mobilité internationale des capitaux n'est pas, non plus, défini sans ambiguïté. Feldstein fait la conjecture que $\beta$ se comporte comme l'intuition le suggère, c'est-à-dire est une fonction croissante de cette mobilité.

L'article fondateur de FH (1980) portait sur 16 pays industrialisés. Les ratios d'investissement et d'épargne étaient des moyennes quinquennales

1 Dans ce modèle, les niveaux de l'investissement brut IB, de l'épargne SB et des entrées nettes de capitaux (EK = IB - SB) dépendent du taux d'intérét intérieur et d'un choc exogène spécifique. Feldstein suppose que l'épargne et l'investissement ne dépendent pas d'une autre variable (le niveau du revenu par exemple) et l'absence d'eftet du taux d'interêt extérieur, une hypothèse difficilement acceptable, même si on suppose que les économies sont de petite taille. 
sur la période 1960-74. Pour chacune des trois sous-périodes, le coefficient $\beta$, qui mesure la fraction de la variation exogène d'épargne restant dans le pays (appelé par la suite «coefficient de rétention d'épargne " (Feldstein et Bachetta (1991)), apparaît de l'ordre de 0,85 à 0,95 et est non significativement différent de l'unité. Les auteurs concluent que 85 à $95 \%$ de l'épargne nationale est investie dans le pays d'origine de cette épargne et rejettent l'hypothèse de parfaite mobilité du capital. L'article de 1980 était court, peu argumenté, sans justification théorique explicite, même approximative, mais établissait de manière indiscutable qu'il n'existe aucune preuve économétrique de l'indépendance des taux d'épargne par rapport aux taux d'investissement.

Une première vague de travaux a cherché à améliorer les estimations en prenant plus sérieusement en compte les problèmes posés par les mécanismes d'ajustement de la balance courante (mécanismes qui se traduisent par une force de rappel à l'égalisation de l'épargne et de l'investissement). Les résultats de Sachs $(1981,1983)$ montrent que le capital est très mobile à l'échelle internationale à la différence des résultats de FH qui montrent que le capital n'est pas mobile. Mais la ligne de recherches ouverte par Sachs n'a curieusement pas été poursuivie et ce n'est que récemment que le problème posé par la tendance à l'équilibre de la balance courante (qui est la face duale de l'équilibre entre l'épargne et l'investissement) va être repris.

Durant les années quatre-vingt et le début des années quatre-vingtdix, les estimations vont se multiplier et confirmer la robustesse des résultats à la FH. Feldstein et Bachetta (1991) actualisent les estimations sur un échantillon de 33 pays de l'OCDE couvrant la période 1974-1986 et parviennent à des résultats assez proches des résultats initiaux. Une actualisation similaire est présentée dans Obsfeldt (1995) qui souligne que $\beta_{C}$ est paradoxalement plus élevé pour la période 1986-90 que pour la période 1981-85. L'article de Tesar (1991) est un article de synthèse qui montre que la corrélation positive élevée est un résultat robuste sur des échantillons plus ou moins larges concernant les pays de l'OCDE.

Une série de fausses notes se fait toutefois entendre dans ce concert d'unanimisme. Citons, outre Sachs dont les travaux n'ont malheureusement pas eu d'écho (Sachs (1981, 1983)), Backus, Kehoe et Kydland (1992) et Taylor (1994) qui obtiennent des $\beta$ ou des corrélations investissement-épargne beaucoup plus faibles qu'à l'habitude. Manifestement, un besoin de clarification économétrique s'impose. C'est une des raisons d'être de cet article.

La dynamique de la recherche de ces dernières années est toutefois dominée par la double idée de la robustesse des résultats $\mathrm{FH}$ et de la pertinence de la problématique $\mathrm{FH}$, autrement dit par la volonté de trouver une issue au puzzle dans le cadre même de cette problématique. Fallait-il envisager son abandon?

Cette question est évidemment centrale. Moosa (1997) a cherché à « résoudre le puzzle Feldstein-Horioka » en faisant intervenir l'idée que les corrélations entre les taux d'investissement et d'épargne ne peuvent rendre 
compte de la mobilité internationale des capitaux car elles ne concernent que les fiux nets d'entrées et de sorties de capitaux, et non des flux bruts: à un même solde correspondent à l'évidence des niveaux extrêmement différents en termes bruts. Et l'intuition suggère que ces mouvements bruts ont fortement augmenté dans la dernière période si on se réfère aux transactions sur les marchés des changes dans une optique de court terme (Berdot, Kébabdjian, Léonard (1999)). Moosa souligne que l'indicateur de FH n'est pas une mesure pertinente de la mobilité internationale des capitaux et que l'augmentation des flux bruts témoigne d'un accroissement de cette mobilité. Néanmoins, au-delà du fait qu'il n'existe aucune statistique systématique et fiable sur les mouvements bruts, il n'est pas possible de rejeter d'un revers de main la pertinence de l'indicateur FH.

En effet, si la mobilité internationale des capitaux ne s'évaluait pas in fine à travers les taux d'épargne et d'investissement, c'est-à-dire en termes nets, les mouvements de capitaux seraient fictifs et $n$ 'auraient assuré aucune fonction de financement. L'accès au marché international peut être extrêmement aisé et se traduire par une très rapide égalisation des taux d'intérêt à travers le monde. Cette caractéristique microéconomique peut être mesurée par des test de parité des taux d'intérêt. Mais cela ne fournit aucune indication sur l'utilisation de ces potentialités au plan macroéconomique. L'intérêt de l'approche $\mathrm{FH}$ tient précisément au fait qu'elle met en relation la sphère financière avec la sphère réelle et cherche à évaluer la mobilité du capital par rapport à sa finalité qui est une meilleure allocation de l'épargne mondiale. En d'autres termes, le test macroéconomique proposé par FH cherche à évaluer l'efficacité de l'allocation mondiale de l'épargne. C'est le point de vue partagé par presque tous les auteurs qui ont cherché à rendre compte de l'apparente anomalie présentée par les tests $\mathrm{FH}$ et c'est le point de vue adopté dans cet article.

\subsection{La deuxième vague : expliquer les corrélations investissement-épargne}

Les premiers tests de la mobilité internationale des capitaux, qui reposaient sur la corrélation entre l'épargne et l'investissement, ont suggéré que le capital ne circulait pas librement, même entre les pays les plus industrialisés. La deuxième vague de travaux cherche à montrer que ces tests sont défectueux parce que les covariances investissement-épargne ne sont pas nécessairement indicatives d'une immobilité internationale du capital.

Nous laisserons de côté une série d'arguments non validés économétriquement, comme l'argument de l'inégalité des taux d'intérêt réels avancé par Frankel $(1986,1993)$ ou l'argument de l'autofinancement des entreprises (Obsfeldt (1995), Obsfeldt et Rogoff (1996) p. 156). 


\subsubsection{Le problème d'endogénéité et de simultanéité}

Une raison évidente de la corrélation est la nature fortement pro-cyclique de l'investissement et de l'épargne, même si on les exprime par rapport au PIB. $\mathrm{Si}$ un choc exogène arrive à affecter les deux variables simultanément, non seulement on ne pourra attribuer la corrélation à une faible mobilité du capital mais cette corrélation pourra être très élevée. C'est pour cette raison que FH (1980) ont restreint leur analyse aux données en coupes transversales de préférence aux séries temporelles car ces dernières sont beaucoup plus sensibles au phénomène de simultanéité. Pour les séries temporelles, différentes tentatives ont été menées pour les ajuster de façon à faire disparaître cet effet pro-cyclique : Sachs (1981), Frankel (1985), Obsfeldt (1990) cherchent à voir ce qui se passe en éliminant l'effet du cycle. Les résultats obtenus ne modifient pas les conclusions principales, à savoir que la corrélation entre les taux d'investissement et d'épargne reste élevée.

Une version complémentaire du problème est que, pour les séries en coupes transversales, les taux d'investissement et d'épargne dépendent du taux de croissance du revenu national (croissance de la population et/ou de la productivité). Cela explique que des études aient cherché à prendre en compte le taux de croissance comme variable supplémentaire dans les ajustements. Mais le résultat a été que, même en gardant constant le taux de croissance (de même que lorsque l'on maintient le cycle constant), le coefficient $\beta$ ne diminue pas (Summers (1985)).

L'aspect le plus connu du problème de l'endogénéité est que le gouvernement réagit de façon systématique aux déséquilibres de la balance des opérations courantes pour tenter de les annuler ou de les limiter. Par exemple, si le gouvernement réagit au déficit commercial induit par une croissance de l'investissement en diminuant les dépenses publiques ou en augmentant les impôts, alors l'investissement et l'épargne intérieurs seront corrélés pour des raisons qui n'ont rien à voir avec la mobilité du capital. L'argument de la « réaction politique " (la politique macroéconomique répond aux changements exogènes pour atteindre un objectif de balance extérieure) a été présenté par Fieleke (1982), Tobin (1983), Westphal (1983), Caprio et Howard (1984), Summers (1985) et Artis et Bayoumi (1989). Summers a appelé cet argument l'hypothèse de «l'équilibre extérieur maintenu ». Artis et Bayoumi (1989) montrent qu'il y a peu de preuves pour considérer que le gouvernement mène une politique budgétaire visant à combattre les déséquilibres de la balance courante, mais que des indices existent pour considérer que la politique monétaire peut avoir un tel objectif.

Il est important de réaliser que l'argument de l'endogénéité a une valeur générale comme le montre Obsfeldt (1986) dans un travail resté presque aussi célèbre que celui de FH. N'importe quelle variable qui influence le taux d'investissement (en plus du coût du capital) sera probablement corrélée avec le taux d'épargne, au moins de façon conjoncturelle. La plupart des chocs introduisent une corrélation positive, mais certains peuvent produire une corrélation négative. Par exemple, un allégement de fiscalité sur l'in- 
vestissement peut contribuer à accroître l'investissement tout en diminuant de façon presque certaine les surplus budgétaires, donc l'épargne intérieure. Ce canal d'endogénéité établit une relation de signe négatif entre l'investissement et l'épargne. On retrouvera dans les estimations économétriques présentées plus loin quelques cas de pays vérifiant de façon structurelle ce type de corrélation, c'est-à-dire pour lesquels la pente de la droite de régression est négative (le cas typique est celui de l'Irlande). Quoi qu'il en soit du signe, il est clair que l'épargne, l'investissement et le solde de la balance courante sont tous endogènes à court terme et on doit s'attendre à une certaine covariance entre eux. Ce point doit être considéré comme un acquis mais il n'est nullement de nature à résoudre le puzzle. Deux questions demeurent:

- Pour les régressions en séries temporelles, en admettant la validité de l'hypothèse de covariance fondée sur l'endogénéité, il reste à expliquer pourquoi il y a si peu d'indépendance entre les variables dans la plupart des économies ouvertes (des corrélations proches de l'unité sur certaines périodes) et pourquoi on observe, comme on le verra avec les données statistiques sur données temporelles, n'importe quelle valeur pour les pentes des droites de régression (positive, négative, inférieure ou supérieure à l'unité), donc une disparité significative dans les relations entre les taux d'épargne et les taux d'investissement.

- La seconde question est à nos yeux plus fondamentale puisqu'elle concerne les régressions en coupes transversales, les plus significatives pour l'analyse de la mobilité internationale des capitaux. L'argument de l'endogénéité et de la covariance, s'il est fort pour les données chronologiques, est de moindre portée sur les données en coupes. Dans ce dernier cas, il n'est pas absurde de considérer que les taux d'épargne sont des variables exogènes dans la relation avec les taux d'investissement du point de vue des différences internationales. Ce point mérite quelques précisions car il constituera une des bases de notre analyse future.

La théorie économique montre que les différences des taux d'épargne entre les pays peuvent s'expliquer par les disparités des comportements des groupes sociaux et des structures de populations. C'est cette distribution à caractère « extra-économique » qui intervient implicitement en amont des ajustements en coupes transversales. Il est alors légitime de considérer que les taux d'épargne sont des variables exogènes et que les taux d'investissement (et donc les flux nets de capitaux entre pays) sont les variables endogènes de la régression.

On trouvera des arguments fondant cette hypothèse dans la littérature récente. Ainsi, au terme d'une étude très complète, Higgins (1998) met en évidence l'influence décisive des données socio-démographiques et arrive à la conclusion que la disparité des taux d'épargne entre pays est exogène par rapport aux taux d'investissement et aux déséquilibres de la balance courante. « Le lien qui va de la démographie aux taux d'épargne, à l'investissement et aux flux d'entrées et de sorties de capitaux est de façon évidente très robuste : les variables démographiques demeurent significatives au seuil 
de $1 \%$ sous n'importe quelle variante, et cela sans changement qualitatif dans les coefficients de distribution par âge » (Higgins, p.362).

Par la suite, dans les régressions en coupes transversales, nous choisirons explicitement de considérer les taux d'épargne comme les exogènes de la relation. Il est encore possible, à la suite de $\mathrm{FH}$, de considérer les taux d'investissement et d'épargne en moyennes quinquennales de façon à éliminer un éventuel effet conjoncturel.

\subsubsection{Le problème de l'ouverture}

Un ensemble de travaux a cherché à montrer que la forte corrélation empirique entre taux d'investissement et d'épargne s'expliquait (notamment pour les séries temporelles) par le fait que les économies présentent une structure duale: une partie de l'activité économique est proche de l'économie fermée et une autre partie se conforme aux caractéristiques d'une économie ouverte et est seule soumise aux effets de l'accroissement de la mobilité internationale des capitaux. Ces travaux ont cherché à rendre compatibles l'observation d'un $\beta$ relativement élevé avec l'hypothèse d'une forte mobilité internationale des capitaux.

Un premier groupe met l'accent sur la présence de biens « échangeables » et de biens « non échangeables » ainsi que sur l'existence de facteurs immobiles. Murphy (1986), Engel et Kletzer (1987), Tesar (1990) et Wong (1990) explicitent sur le plan analytique comment l'investissement peut être limité par l'offre d'épargne intérieure lorsque ces éléments sont introduits et rendent compte sur cette base de la corrélation empirique entre taux d'investissement et taux d'épargne. Malgré le caractère apparemment constructif de ce résultat, l'explication ouvre la porte à de nouvelles anomalies.

En effet, un des faits les mieux établis des recherches économétriques est qu'il existe une disparité dans les corrélations entre les taux d'investissement et les taux d'épargne par groupes de pays selon leur degré de développement. Les pays industrialisés, si on les étudie en séries chronologiques, ou l'ensemble des pays industrialisés, si on les étudie en coupes transversales, se caractérisent par une corrélation beaucoup plus forte (en termes de $\beta$ et de $R^{2}$ ) que les pays en développement. Cela suggère que le capital circule moins librement entre les grands pays industrialisés qu'entre pays en développement : paradoxalement, la globalisation financière serait plus importante pour les seconds que pour les premiers.

Une variante du problème de l'ouverture est l'effet lié à la taille des pays (Harberger (1980)). Cet effet introduit un biais automatique qui résulte du fait qu'un grand pays est plus proche d'une économie fermée qu'un petit. La faible corrélation entre les taux d'investissement et d'épargne à l'échelle « régionale » a été vérifiée (Bayoumi et Rose (1993), par exemple). Ce fait empirique évident ne permet pas de soutenir que les marchés financiers sont moins bien intégrés au plan national qu'ils ne le sont au plan «local ». De même au plan international, un grand pays peut financer une large partie 
de ses investissements à l'aide de son épargne intérieure sans avoir besoin de recourir à l'épargne extérieure.

Une version plus sophistiquée de l'effet de taille concerne le problème des externalités. La validité des tests à la $\mathrm{FH}$ repose sur l'hypothèse que les variables externes sont exogènes, notamment le taux d'intérêt international. Cette hypothèse peut être acceptée dans le cas des «petits pays » mais n'est plus vérifiée pour les « grands pays » qui ont la taille suffisante pour influencer les variables extérieures. Les externalités produites par une variation du taux d'épargne conduisent alors à établir une corrélation avec le taux d'investissement, même en présence d'une parfaite mobilité du capital. Pour justifier la significativité de $\beta$ en termes de mobilité du capital, il faut supposer que le taux d'intérêt extérieur est exogène, hypothèse qui n'est pas respectée lorsque l'on considère des grands pays (Tobin (1983), Obsfeldt (1986)). On peut alors montrer que, pour une sensibilité de l'investissement au taux d'intérêt et une mobilité du capital identiques, $\beta$ sera vraisemblablement plus élevé dans les grands pays que dans les petits pays.

Sachs (1983) confirme cet effet et montre que le taux de déficit extérieur par rapport au PIB est négativement corrélé avec la taille du pays. De plus, l'inclusion des petits pays (en termes de PIB) affaiblit les corrélations investissement-épargne (Murphy (1984)). Murphy considère un échantillon de 17 pays de l'OCDE et trouve que le groupe des 10 grands pays se caractérise par un coefficient $\beta$ de 0,98 alors que celui des 7 petits pays est de 0,59 . On retrouve une telle disparité entre pays industrialisés et pays en développement (Dooley, Frankel et Mathieson (1987)). Toutefois la correction du biais introduit par l'effet de taille laisse persister une corrélation élevée entre les taux d'investissement et d'épargne; les résultats dégagés par $\mathrm{FH}$ ne proviennent donc pas d'un artefact lié à ce biais (Fieleke (1982), Tesar (1991)). Notons, par ailleurs, que ces résultats sont difficiles à interpréter en termes de mobilité internationale des capitaux car ils reposent sur une comparaison de groupes de pays (grands et petits pays industrialisés; pays industrialisés et pays en développement) supposés artificiellement appartenir à des ensembles disjoints. Les groupes de pays constituent, en fait, tous les sous-ensembles du même système financier international et, par conséquent, il est nécessaire de comparer les degrés de mobilité obtenus lorsque l'on distingue non pas des groupes différents de pays mais des niveaux inclusifs croissants du même ensemble de départ («poupées russes »).

C'est pourquoi, dans l'étude économétrique qui va suivre, nous serons amenés à définir plusieurs niveaux d'extension : le cercle des grands pays industrialisés; le cercle des pays industrialisés (dont le cercle précédent est un sous-ensemble); le cercle plus large des pays industrialisés et des pays émergents (dont les cercles précédents sont des sous-ensembles). La prise en compte d'un quatrième cercle plus extensif ne se justifie pas car les mouvements de capitaux deviennent, au-delà du troisième cercle, pratiquement inexistant. La comparaison des degrés de mobilité en coupes transversales se fera ainsi entre résultats concernant des niveaux inclusifs croissants du système financier international. 


\subsubsection{Le problème de l'explicitation du modèle structurel}

Les équations de régression, qui ne sont pas explicitement dérivées d'une théorie, ne permettent pas d'évaluer la signification du coefficient de corrélation ou du terme $\beta$. Beaucoup de modèles, $y$ compris les modèles de croissance néoclassiques ou les modèles de cycles réels, exhibent des propriétés de corrélation étroite entre l'investissement et l'épargne quel que soit le degré de mobilité du capital.

Grinols (1996) et Shibata et Shintani (1998) montrent comment le modèle de croissance néoclassique en univers stochastique s'accorde avec les corrélations observées entre l'investissement et l'épargne, même en présence d'une parfaite mobilité des capitaux. Finn (1990) établit l'existence de relations dynamiques variées (positive ou négative) entre épargne et investissement dans une petite économie sous l'influence de chocs technologiques. Ces modélisations ne concernent toutefois qu'une petite économie ouverte, aucune externalité n'étant prise en compte.

Le modèle néoclassique à deux pays de Baxter et Crucini (1993) fait exception en ce qu'il cherche à prendre en compte les interdépendances internationales. Ils montrent, dans un contexte de parfaite mobilité des capitaux réels et financiers, qu'il existe une corrélation positive entre les taux d'investissement et d'épargne et que les corrélations observées ne sont pas contradictoires avec les prédictions du modèle. De plus, le modèle permet de prédire que les corrélations doivent être plus élevées pour les grands pays que les petits pays, conformément à l'observation empirique déjà signalée.

Le facteur explicatif de leur corrélation épargne-investissement est en vérité très simple : il tient aux effets synchrones sur l'épargne et l'investissement des chocs qui affectent l'économie. En d'autres termes, le problème de l'identification du modèle structurel n'est rien d'autre qu'une variante plus sophistiquée du problème de l'endogénéité et de la simultanéité.

Les résultats obtenus, aussi intéressants soient-ils, ne relèvent pas directement de l'analyse de la mobilité internationale des capitaux. En effet, le fait d'expliciter les mécanismes par lesquels un modèle macroéconomique est, avec un niveau élevé de mobilité du capital, capable de prédire les corrélations observées entre l'épargne et l'investissement ne dit pas que les observations sont effectivement dues à ces mécanismes. En second lieu, l'effort d'explicitation du modèle porte sur une seule économie; l'approche est une approche-pays : les études économétriques sont relatives aux séries temporelles et les corrélations investissement-épargne ne concernent pas le système financier international pris comme un tout, démarche qui exige une étude en coupes transversales. En d'autres termes, les travaux recensés sont de peu d'utilité pour une analyse de la mobilité internationale du capital. 


\subsubsection{Le problème de la contrainte d'équilibre de la balance courante}

Le dernier problème d'interprétation posé par la forte corrélation entre les taux d'investissement et d'épargne est celui de la tendance à l'annulation du solde de la balance courante (donc des flux nets de capitaux). On peut penser que la corrélation exprime simplement le jeu d'une «contrainte de solvabilité à long terme de la balance courante ». Qu'en est-il ?

Ghosh (1995) montre que les séries temporelles d'épargne et d'investissement ne sont pas stationnaires, ce qui semble indiquer l'absence d'une tendance à l'équilibre de la balance courante. Il tire d'ailleurs de ce résultat l'idée que le coefficient de corrélation serait sans signification car: ou bien (a) l'épargne et l'investissement sont co-intégrés, auquel cas leur corrélation asymptotique est l'unité; ou bien (b) ils ne sont pas co-intégrés, auquel cas leur corrélation est asymptotiquement nulle. C'est la raison pour laquelle Ghosh propose d'utiliser des mesures alternatives de la mobilité du capital comme le critère de la consommation (qui donne des résultats satisfaisants en montrant que la mobilité du capital a effectivement augmenté dans la période récente).

Coakley, Kulasi et Smith (1996) reprennent cette question et établissent curieusement le résultat exactement inverse de celui de Ghosh : les taux d'épargne et d'investissement apparaissent intégrés d'ordre 1. La raison explicative de la forte corrélation entre l'investissement et l'épargne ne serait pas un puzzle mais tiendrait à un artefact statistique lié à la régression. Ils recommandent donc d'utiliser un autre critère, celui du solde de la balance courante rapporté au PIB (qui est identiquement égal à la différence entre les taux d'investissement et d'épargne). Ce solde devrait être intégré d'ordre 0 si les mouvements de capitaux étaient les projections passives des déséquilibres de la balance de base et si les ajustements de la balance des paiements étaient efficaces (contrainte de solvabilité). Leur étude économétrique confirme l'hypothèse de stationnarité pour le solde de la balance courante rapporté au PIB puisque les taux d'épargne et d'investissement sont co-intégrés d'ordre 1. La régression $\mathrm{FH}$ capturerait pour eux la contrainte de solvabilité à long terme de la balance extérieure plutôt que le degré de mobilité du capital.

Dans un travail ultérieur, Coakley, Hasan et Smith (1999) montrent que, par rapport aux pays de l'OCDE, le test $A D F$ rejette généralement l'hypothèse de stationnarité du solde courant pour les pays en développement $^{2}$, ce qui tend à prouver que les pays en développement sont plus sensibles aux chocs externes; en d'autres termes, l'impact des chocs sur la balance courante a des effets cumulatifs. Le résultat permet d'expliquer la faible corrélation observée entre l'investissement et l'épargne dans le cas de pays en développement (et plus généralement dans le cas des petits pays) par rapport aux pays de l'OCDE. Cette observation, qui est difficilement

2 L'hypothèse de processus stationnaire n'est pas rejetée pour 3 pays en développement seulement. 
explicable à partir de l'argument de la mobilité des capitaux, devient facilement interprétable : les pays en développement se caractérisent par une contrainte de solvabilité relâchée (due aux apports importants de capitaux extérieurs liés à l'aide), relâchement qui se repère par un niveau de dette extérieure souvent supérieur à celui qui assurerait sa stabilité.

Les tests de racine unitaire, associés à l'idée qu'il existe une contrainte de solvabilité à long terme de la balance courante, semblent invalider l'utilisation de méthodes à la FH. Toutefois, ces résultats ne sont pas suffisants pour nous faire rejeter une approche en termes de régression des taux d'investissement sur les taux d'épargne. En effet, le nombre de données utilisées pour établir que les séries d'épargne et d'investissement sont stationnaires est trop faible (18 données annuelles chez Ghosh; 30 chez Coakley, Kulasi et Smith) pour que les résultats soient significatifs. De plus, ces tests concernent des séries temporelles par pays et non des données en coupes transversales, domaine principal d'investigation pour analyser le système financier international. Les deux domaines n'ont pas de relation directe et, comme on le précisera plus loin, exhibent des propriétés de nature très différente. L'un accorde une place primordiale à la contrainte de solvabilité et aux mécanismes d'équilibrage de la balance extérieure (séries temporelles); l'autre est beaucoup plus sensible aux effets de la mobilité internationale du capital (données en coupes). Cette distinction est à la base de nos investigations.

\section{$3 \quad$ Retour sur les tests habituels et mise en cause des idées reçues}

L'étude empirique de cette section a pour but de montrer que les tests traditionnels fondés sur la corrélation entre taux d'investissement et taux d'épargne sur données temporelles ou sur coupes transversales n'invalident pas, contrairement aux idées reçues en ce domaine, la baisse du paramètre ( et donc la réalité d'un mouvement de globalisation financière.

L'échantillon retenu est de 31 pays $^{3}$ représentant plus de $85 \%$ du produit mondial : ils ont été choisis en fonction de leurs fortes participations à ce produit et de la disponibilité de données permettant la constitution

3 Les 31 pays sont les suivants (entre parenthèses, on mentionne les abréviations retenues, qui sont généralement celles de la Banque mondiale, pour les tableaux de résultats statistiques): Argentine (ARG). Afrique du Sud (ZAF), Allemagne (DEU), Australie (AUS), Autriche (AUT), Belgique (BEL), Brésil (BRA), Canada (CAN), Chili (CHL), Corée (KOR), Danemark (DNK), Espagne (ESP), États-Unis (USA), Finlande (FIN), France (FRA), Grèce (GRC), Indonésie (IDN), Irlande (IRL), Italle (ITA), Japon (JPN), Luxembourg (LUX), Mexique (MEX), Norvège (NOR), Nauvelle-Zélande (NZL), Pays-Bas (NLD), Portugal (PRT), RoyaumeUni (GBR), Singapour (SGP), Suède (SWE), Suisse (CHE), Thailande (THA). Le G7 recoupe la définition habituelle (pays notés en gras). G22 correspond au G7 auquel ont été rajoutés 15 pays industrialisés (pays notés en caractère normal). Le passage de G22 à G31 résulte de la prise en compte des pays émergents (pays notés en italique). La différence entre G7 et G22 est donc principalement fondée sur un critère de taille; la différence entre G22 et G31 sur un critère de développement. On notera que les G7, G22 et G31 sont construits par extensions successives; il en est de même du G15, G22 et G31. 
d'un ensemble homogène sur une période relativement longue (1965-1996). Ces 31 pays forment le groupe noté G31, où coexistent pays industrialisée et pays émergents. Trois sous-ensembles ont été isolés : le groupe des 7 pays les plus riches (ou G7), l'Europe des 15 (ou G15) et enfin un groupe de 22 pays industrialisés ${ }^{4}$.

\subsection{En pooling}

Les régressions en pooling, dans la mesure où elles cherchent à apprécier le mouvement de long terme du coefficient $\beta$, sont fondées sur l'utilisation d'une variable muette. Celle-ci vaut 1 de 1980 à 1996 si l'on considère, comme il est d'usage, que cette date marque une rupture dans l'intensité de la circulation internationale des capitaux. La présence de la variable notée DU80 a pour but de tester l'évolution de la relation entre investissement et épargne domestiques en liaison avec le mouvement de libéralisation et d'innovation financières. On ne saurait en outre ignorer que parmi les facteurs qui ont pu contribuer à l'affaiblissement de la corrélation entre investissement et épargne après 1980 figure l'effet d'assainissement des finances publiques : dans le cas des économies européennes, afin de respecter les critères de convergence préparant à la monnaie unique; dans le cas des PVD et des économies en transition, afin de satisfaire aux programmes d'ajustement structurel $^{5}$. La diminution de la désépargne de l'État a pu s'assortir d'une diminution du taux d'épargne domestique sans effet clair sur le taux d'investissement.

Cependant, la variable muette ne veut pas traduire l'effet d'un " break » structurel (qui n'a pas été estimé) mais doit aider à vérifier la stabilité temporelle de la relation. L'idée de « rupture » est d'autant moins pertinente que notre analyse évalue surtout la relation entre les valeurs des paramètres $\alpha$ et $\beta$ obtenues sur coupes transversales, et ce année après année.

L'équation testée est donc pour chacun des groupes retenus :

$$
i_{j t}=\alpha+\beta s_{j t}+\alpha_{1} D U 80+\beta_{1} s_{j t} D U 80
$$

Les variables sont les taux d'investissement et d'épargne domestiques bruts des pays $j$ du groupe étudié pour l'année $t$. On notera que les taux d'épargne et d'investissement sont exprimés en pourcentage, de sorte qu'il en sera de même de la constante de la régression.

Pour les 3 premiers groupes (les plus homogènes) la réduction du paramètre $\beta$ est fortement significative. La valeur du paramètre $\beta_{1}$, significativement négative, mesure cette diminution de la pente de la relation

4 Pour le G31 et le G22, les données disponibles relatives aux taux d'épargne et d'investissement bruts sont celles de la Banque mondiale (disponibles de façon homogène depuis 1965). Pour les groupes G7 et G15, les données (source: Union européenne) peuvent étre disponibles depuls 1960.

5 Nous remercions le rapporteur anonyme quil nous a suggéré la mise en jeu de ce dernier mécanisme, d'autant plus significatif que le taux de déficit public était initialement important. 
Tableau 1 : tests en pooling

\begin{tabular}{|cccccc|}
\hline Pooling & $\alpha$ & $\beta$ & $\alpha_{1}$ & $\beta_{1}$ & $\mathrm{R}^{2}$ \\
G7 (1960-1996) & 1.59 & 0.86 & 9.21 & -0.12 & 0.90 \\
& $(2.90)$ & $(41.48)$ & $(4.26)$ & $(-3.61)$ & \\
G15(1960-1999) & 14.56 & 0.37 & 2.87 & -0.23 & 0.35 \\
& $(10.40)$ & $(6.86)$ & $(1.82)$ & $(-3.57)$ & \\
G22 (1965-1996) & 10.88 & 0.60 & 1.37 & -0.17 & 0.43 \\
& $(7.50)$ & $(10.28)$ & $(0.78)$ & $(-2.35)$ & \\
G31 (1965-1996) & 9.83 & 0.64 & -2.78 & 0.03 & 0.51 \\
& $(9.82)$ & $(15.56)$ & $(-2.07)$ & $(0.47)$ & \\
\hline
\end{tabular}

Les taux et les paramètres sont exprimés en pourcentage. Les $t$ de Student sont donnés entre parenthèses. Ils sont corrigés de l'hétéroscédasticité (procédure de White). La même procédure est appliquée à l'ensemble des tableaux qui suivent.

entre $i$ et $s$. L'introduction dans l'échantillon de pays émergents (passage au groupe G31) révèle un mouvement en sens inverse, mais cette hausse n'est pas significative. Les régressions sur pooling valident donc la diminution de l'indicateur $\beta$ sur toute la période.

\subsection{En séries temporelles}

Les régressions sur données temporelles de la forme :

$$
i_{t}=\alpha+\beta s_{t}
$$

effectuées pour chacun des 31 pays sur toute la période (1965-1996), puis en distinguant les deux sous-périodes (1965-1979 et 1980-1996), confirment généralement le phénomène de baisse du coefficient $\beta^{6}$ : il se produit pour 21 pays sur les 31 . Mais les valeurs prises par ce coefficient sont parfois aberrantes eu égard à l'interprétation qu'en donnent FH. Le domaine de

6 Les régressions (sur données temporelles ou sur coupes transversales) entre $i$ et $s$ ne cherchent pas à spécifier une fonction pour $i$ mais à s'interroger sur la nature et les implications d'une démarche de " mise en correspondance " d' $i$ et $s$, "à la FH ". Cela étant, les tests traditionnels de spécification peuvent ne pas être inutiles. Encore faut-il qu'ils soient possibles et pertinents. Le nombre de degré de liberté des régressions est ici torcément faible (analyses annuelles sur les périodes 1960/96 ou 1965/96, analyses transversales pour $7,15,22$ ou 31 pays). L'accent mis sur les régressions en coupes transversales réduit l'intérêt des analyses d'autocorrélation éventuelle des résidus, interdit de passer sur le compte de " points aberrants " ce qui n'est que la marque de caractéristiques différentes entre pays, et rend moins cruciai le débat endogénéité/exogénéité de l'épargne. II n'était pas possible de conduire des tests d'exogénéité à la Hausman, ou à la Davidson et MacKinnon, qui auraient requis l'intégration de variables instrumentales et la spécification d'une fonction d'épargne.

En revanche, les tests de causalité ont été effectués et les résultats peuvent justifier notre hypothèse d'exogénéité de l'épargne: sur les 31 pays retenus (au seuil de $5 \%$ ), alors que i cause s au sens de Granger dans seulement 4 cas, s cause i dans 12 cas et meme 14 au seuil de $6 \%$. Enfin, les estimateurs ont été corrigés pour l'hétéroscédasticité.

Les résultats détaillés de nos estimations (sur séries temporelles, coupes périodiques et instantanées) sont fournis sur demande à l'adresse e-mail : Jean-Pierre.Berdot@ univ-poitiers.fr 
définition prévu par leur analyse (de 1 à une valeur faiblement positive au fur et à mesure que devrait s'opérer la croissance de la mobilité) est clairement invalidé. L'indicateur $\beta$ peut être négatif (Allemagne, Royaume Uni), le cas le plus atypique étant celui de l'Irlande dont le paramètre évolue dans le sens prévu par $\mathrm{FH}$ mais en passant d'une valeur de 0.58 à une valeur de -0.60 ! Le coefficient $\beta$ peut aussi être supérieur à l'unité (Autriche, France, Suisse), le cas extrême en la matière étant celui de la Finlande (dont le paramètre se réduit certes, mais de 1.47 à 1.34 !).

Tableau 2 : Estimations des $\beta$ sur séries temporelles

\begin{tabular}{|c|c|c|c|}
\hline \multicolumn{3}{|c|}{ Classement des pays selon les valeurs de $\beta$ obtenus sur séries temporelles sur la période 1965-1996 } \\
\hline$\beta<0$ & $\beta=0$ & $0<\beta<1$ & $\beta>1$ \\
\hline IRL & LUX, & ARG, AUS, BEL, BRA, CAN, CHL, DEU, GBR, & AUT, CHE, DNK, ESP, FIN, \\
& MEX, NOR & GRC, IDN, KOR, NZL, PRT, SGP, USA, ZAF & FRA, ITA, JPN, NLD, SWE, THA \\
\hline
\end{tabular}

En réalité, ces résultats apparemment aberrants peuvent être interprétés par une réécriture de la relation de FH. L'investissement domestique brut I est assurée par l'épargne intérieure brute $S$ et un apport de l'épargne extérieure sous forme d'entrées nettes de capitaux $K$, soit : I $=S+K$. Il suffit de diviser par le PIB pour exprimer la relation entre taux : $i=s+k$, lorsque $k$ est le taux d'épargne extérieure (ratio des entrées nettes de capitaux positives, ou négatives dans le cas de sorties de capitaux). On a donc:

$$
k=i-s=\alpha+(\beta-1) s
$$

L'indicateur $\beta$, peut alors être facilement réinterprété. Une valeur de $\beta$ égale à l'unité n'implique pas nécessairement une situation d'immobilité internationale des capitaux, soit ici la nullité des entrées nettes de capitaux $\mathrm{K}$; elle traduit simplement l'indépendance des apports extérieurs vis-à-vis de l'épargne intérieure: le taux $k$ est alors autonome et s'identifie au paramètre $\alpha$ qui doit être interprété comme la part des apports de capitaux qui ne résultent pas d'une épargne domestique préalable. La seconde composante du taux $k$, soit $(\beta-1) s$, mesure par conséquent la part des entrées de capitaux qui dépend, au contraire, des efforts préalables d'épargne (et seule l'existence de cette composante est impliquée par la condition $0<\beta<1$ ).

La proximité (plus ou moins étroite) de $\beta$ par rapport à 1 ne peut donc renseigner sur le degré de mobilité internationale des capitaux : elle rend compte uniquement de la corrélation entre $k$ et $s:$ un $\beta$ inférieur (supérieur) à l'unité signale une covariance négative (positive) entre $k$ et $s$, de sorte qu'une valeur négative de $\beta$ peut se rencontrer.

\subsection{En coupes transversales}

Deux types de tests en coupes transversales ont été conduits : coupes périodiques et coupes instantanées. Les valeurs de $\beta$ y sont comparables et, dans 
les deux cas, les résultats obtenus divergent radicalement de ceux en séries temporelles, ce qui manifeste bien la différence de nature entre les équations relatives aux tests temporels et celles relatives aux tests en coupes.

\subsubsection{En coupes périodiques}

Les tests en coupes périodiques sont fondés sur la relation :

$$
\bar{i}_{j}=\alpha+\beta \bar{s}_{j}
$$

où les variables sont les taux moyens quinquennaux (mesurés entre les années $t$ et $t-4$ ) des pays $j$ du groupe. Pour les groupes G7 et G15, les régressions ont été conduites pour les années de 1964 à 1996 (1964-1999 pour l'Europe des 15) et pour 1969-1996 pour les groupes G22 et G31 en fonction de la disponibilité des données.

Pour chacun des groupes et pour toutes les années, les paramètres $\beta$ sont tous, à la différence des estimations en séries chronologiques, positifs et inférieurs à l'unité. Ils décroissent au cours du temps ${ }^{\top}$ comme on peut s'en assurer à la lecture graphique de l'évolution des $\beta$ tirés de l'élargissement de l'échantillon des pays par le passage aux groupes G15, G22 et G31 (voir graphique 1).

Graphique 1 : Évolution des $\beta$ issus des tests en coupes périodiques (G15, G22, G31)

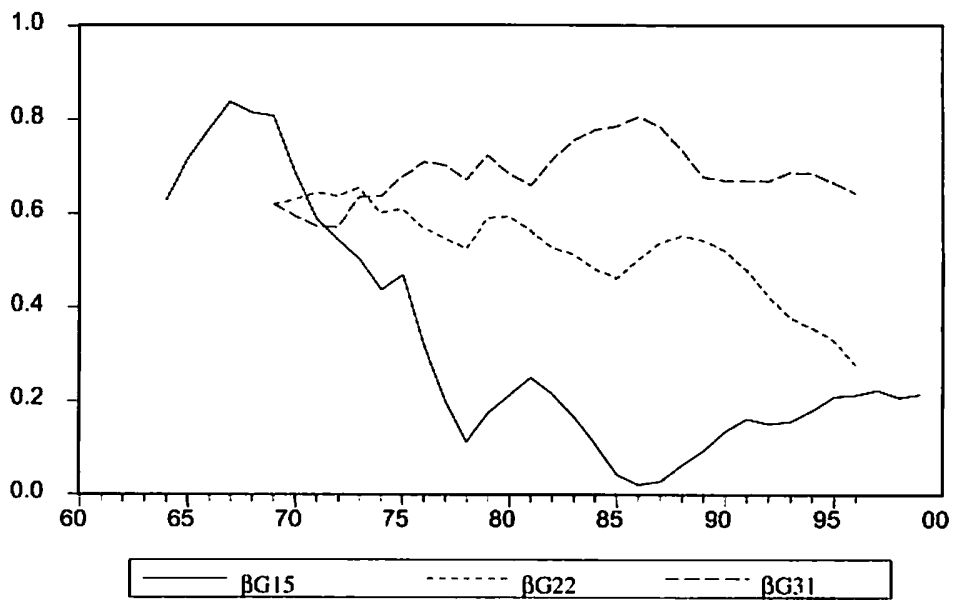

En moyenne, le coefficient $\beta$ du G15 et du G22 chute de la valeur 0,7 au début des années soixante-dix à approximativement 0,2 pour les années quatre-vingt-dix. Seul le G31 (dont font partie les petits pays industrialisés

7 Cette décroissance sera mesurèe plus rigoureusement par la suite à partir des valeurs des paramètres $\beta$ obtenus sur coupes instantanées dont les résultats dupliquent ceux des coupes périodiques. 
non membres de l'Union européenne et les pays émergents) fait apparaître une relative stabilité de $\beta$ autour de la valeur 0,7 . Les observations qui précèdent s'inscrivent donc en faux contre deux idées courantes de la littérature économétrique présentée dans la première section.

Premièrement, à l'opposé de l'idée courante de la défaillance des tests à la FH, nous constatons, pour les pays industrialisés, l'existence d'une très forte tendance à la croissance de la mobilité des capitaux à l'aide des tests standards en coupes périodiques. L'Europe se caractérise, comme il était prévisible, par l'intensité la plus forte; mais l'ensemble de G22 converge vers le même niveau que l'Europe, c'est-à-dire le niveau en principe le plus élevé. En d'autres termes, l'idée que l'Europe se caractériserait comme un espace de mobilité privilégié des capitaux n'est pas validée : la croissance de la mobilité internationale du capital apparaît comme un phénomène mondialisé concernant, de plus en plus, au même degré tous les pays industrialisés.

Deuxièmement, à l'opposé de l'idée courante d'une valeur relativement faible de $\beta$ pour les pays en développement, nous trouvons que ces pays (du moins les pays émergents) sont plutôt caractérisés par un $\beta$ supérieur (rappelons que la différence entre G22 et G31 est fondée sur un critère de développement). De plus, nous observons que le niveau de $\beta$ est plutôt stationnaire en longue période pour G31. Il est vrai que le phénomène d'inversion de la hiérarchie des valeurs de $\beta$ entre les G22 et G31 résulte d'un renversement historique puisque, avant les années soixante-dix, la hiérarchie des valeurs est plutôt conforme à celle généralement retenue dans la littérature. Les trente dernières années mettent donc clairement en évidence, au sens du test $\mathrm{FH}$, un mouvement de divergence entre deux groupes de pays : les pays industrialisés entre lesquels s'est très fortement accrue la mobilité des capitaux et le groupe des pays moins industrialisés qui apparaissent comme les exclus de la globalisation financière. L'existence de clubs de mobilité différentielle est donc étroitement liée au critère de développement.

\subsubsection{En coupes instantanées}

Les tests en coupes instantanées reposent sur une relation de la forme:

$$
i_{j}=\alpha+\beta s_{j}
$$

estimée chaque année pour chaque groupe. Les périodes de régression sont (1960-1996) pour le groupe G7, (1960-1999) pour G15 et (1965-1996) pour les groupes G22 et G31.

Comme pour les coupes périodiques, les paramètres $\beta$ sont positifs (sauf pour le G15 en 1976) et inférieurs à l'unité. On pourra trouver en annexe les résultats complets de ces tests. Un aperçu rapide peut en être donné à l'aide du graphique 2.

Le fait marquant qui ressort du graphique 2 est la tendance générale du paramètre $\beta$ à la baisse pour G15 et G22 et sa stabilité pour G31. Ces comportements confirment les observations effectuées en coupes périodiques. 
Graphique 2: Évolution des $\beta$ issus des tests en coupes instantanées (G15, G22, G31)

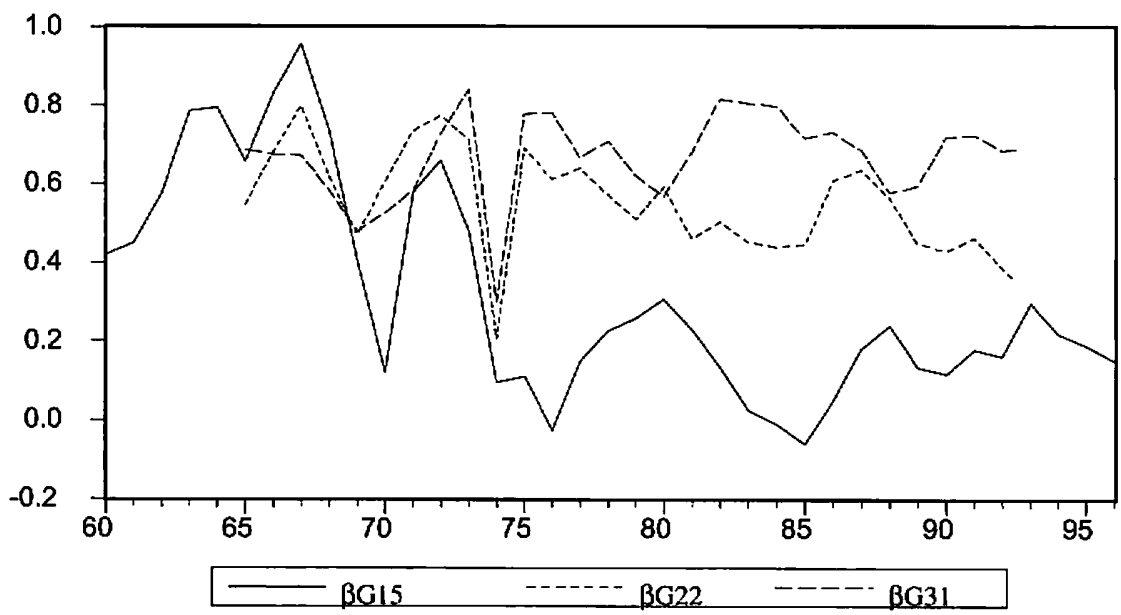

La tendance à la baisse de $\beta$ mérite d'être précisée et mieux justifiée que par la simple observation des graphiques. Pour cela, on a utilisé les valeurs des coefficients $\beta$ estimés sur coupes instantanées ${ }^{8}$ et on a testé une relation de la forme :

$$
\beta_{t}=c+c_{1} D U 80
$$

pour chacun des groupes. Une baisse du paramètre au cours du temps se repèrera par une valeur significativement négative du paramètre affecté à la variable muette périodique (coefficient $c_{1}$ ). Un test parallèle sur les paramètres $\alpha$ a aussi été conduit sous la forme:

$$
\alpha_{t}=d+d_{1} D U 80
$$

Les résultats sont indiqués dans le tableau 3.

Tableau 3 : Comportements temporels des $\alpha$ et $\beta$ pour les 4 groupes

\begin{tabular}{|c|c|c|c|c|}
\hline$\alpha$ & $1960-1996$ & $1960-1996$ & $1965-1996$ & $1965-1996$ \\
\hline & G7 & G15 & G22 & G3I \\
\hline \multirow[t]{2}{*}{$d$} & 0.531 & 12.216 & 10.535 & 9.918 \\
\hline & $(1.21)$ & $(7.47)$ & $(9.79)$ & (10.25) \\
\hline \multirow[t]{2}{*}{$d_{1}$} & 4.190 & 4.840 & 1.429 & -3.040 \\
\hline & $(5.50)$ & (3.09) & $(1.21)$ & $(-2.75)$ \\
\hline $\mathrm{R}^{2}$ & 0.474 & 0.171 & 0.050 & 0.211 \\
\hline
\end{tabular}

\begin{tabular}{|c|c|c|c|c|}
\hline$\beta$ & $1960-1996$ & $1960-1996$ & $1965-1996$ & $1965-1996$ \\
\hline & $G 7$ & \multicolumn{1}{c}{ G15 } & \multicolumn{1}{c}{$G 22$} & $\mathrm{G} 3$ \\
\hline$c$ & 0.908 & 0.463 & 0.611 & 0.641 \\
& $(46.05)$ & $(7.13)$ & $(16.22)$ & $(18.33)$ \\
$c_{1}$ & -0.154 & -0.299 & -0.173 & 0.038 \\
& $(-4.33)$ & $(-4.54)$ & $(-3.55)$ & $(0.95)$ \\
$\mathrm{R}^{2}$ & 0.361 & 0.334 & 0.300 & 0.031 \\
\hline
\end{tabular}

Les paramètres attachés à la variable muette temporelle DU80 sont significativement négatifs pour les trois premières groupes. Pour le G31, qui

8 Les résultats sont de même ordre pour les valeurs des $\beta$ issus de estimations en coupes périodiques 
se caractérise, comme on l'a vu, par une apparente stabilité de $\beta$, le coefficient $c_{1}$ est certes positif mais il n'est pas significatif. Comme on pouvait s'y attendre, le mouvement à la baisse de $\beta$ n'est donc pas invalidé pour G31.

Une dernière observation doit être faite, concernant cette fois le groupe G7. Le graphique 3 présente le $\beta$ relatif à ce groupe.

Graphique 3 : Évolution du $\beta$ issu des tests en coupes instantanées (G7)

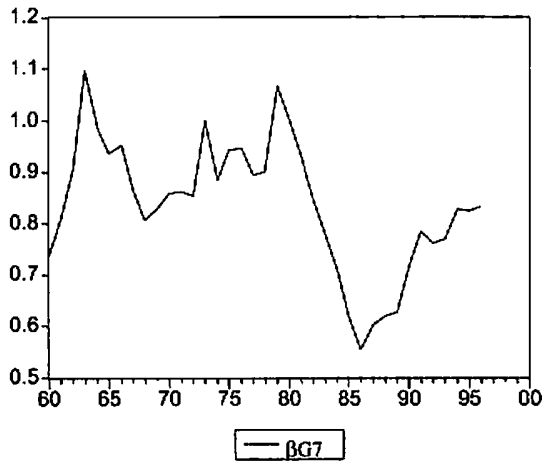

Le G7 se caractérise bien par une baisse de $\beta$ jusqu'en 1985 conformément à la tendance générale des pays industrialisés, mais par une hausse ensuite. La « décorrélation » entre taux d'épargne et d'investissement domestiques s'accélère fortement au cours des premières années de la libéralisation financière (ce qui valide notre distinction des deux sous-périodes et de la variable muette DU80); ce mouvement est suivi d'une apparente « recorrélation » depuis lors qui, au delà de ce que l'on pourrait comprendre comme la correction d'un surajustement, témoigne d'un réaménagement des circuits financiers en rapport avec l'évolution des contraintes de balances de paiements des pays concernés. Cette observation mériterait une analyse fouillée qu'il n'est possible de mener dans le cadre de cet article.

\section{Construction de tests complémentaires et voies nouvelles de recherches}

La mise en évidence de la décroissance sur le long terme de l'indicateur $\beta$ pourrait en première instance conforter le choix de $\mathrm{FH}$ qui en ont fait la mesure macroéconomique unique du degré de mobilité internationale des capitaux. Cependant ces premiers résultats empiriques jettent un doute sur la validité de l'analyse même de FH. Les tests temporels ont fait apparaître des situations atypiques (paramètres $\beta$ supérieurs à l'unité ou négatifs) et les tests en coupes transversales traduisent un comportement fluctuant à court terme des paramètres avec trend à la baisse. Les deux types de régression sur 
la relation linéaire entre taux d'investissement et taux d'épargne amènent surtout à s'interroger sur le comportement, traditionnellement ignoré par la littérature, du paramètre $\beta$ qui est l'ordonnée à l'origine de la droite de régression.

\subsection{L'Hypothèse directrice}

Les tests sur les paramètres issus des régressions de l'équation (8) en coupes instantanées ${ }^{3}$ révèlent une hausse du paramètre (qui est très significative pour les groupes G7, G15) alors que ce paramètre semble décroître pour le groupe G31. Il apparaît donc une possible divergence entre les mouvements respectifs des paramètres $\alpha$ et $\beta$. En outre, pour ces régressions, comme à un moindre degré pour celles qui portent sur les séries temporelles, le coefficient $\alpha$ prend souvent des valeurs négatives, que l'on peut associer à des valeurs élevées du paramètre $\beta$ et notamment à des valeurs proches de l'unité. L'éventuelle relation inverse entre les paramètres $\alpha$ et $\beta$ mérite en conséquence d'être testée.

Pour les quatre groupes, on a testé une relation de la forme:

$$
\beta_{t}=a+b \alpha_{t}
$$

sur l'ensemble de la période et sur les deux sous-périodes.

Tableau 4 : Relations entre $\beta$ et $\alpha$ (G7, G15, G22, G31)

\begin{tabular}{|c|c|c|c|c|c|c|c|}
\hline G7 & $a$ & $b$ & $\mathrm{R}^{2}$ & G15 & $a$ & $b$ & $\mathrm{R}^{2}$ \\
\hline $60 / 96$ & $\begin{array}{c}0.936 \\
(147.46)\end{array}$ & $\begin{array}{c}-0.041 \\
(-29.64)\end{array}$ & 0.941 & $60 / 99$ & $\begin{array}{c}0.939 \\
(47.87)\end{array}$ & $\begin{array}{c}-0.043 \\
(-28.82)\end{array}$ & 0.935 \\
\hline $60 / 79$ & $\begin{array}{c}0.929 \\
(143.50)\end{array}$ & $\begin{array}{c}-0.042 \\
(-12.95)\end{array}$ & 0.870 & $60 / 79$ & $\begin{array}{c}0.946 \\
(112.13)\end{array}$ & $\begin{array}{c}-0.040 \\
(-67.54)\end{array}$ & 0.995 \\
\hline $80 / 96$ & $\begin{array}{c}0.973 \\
(71.54) \\
\end{array}$ & $\begin{array}{c}-0.047 \\
(-19.66) \\
\end{array}$ & 0.958 & $80 / 99$ & $\begin{array}{c}0.721 \\
(10.28)\end{array}$ & $\begin{array}{r}-0.033 \\
(-7.53) \\
\end{array}$ & 0.702 \\
\hline G22 & $a$ & $b$ & $\mathrm{R}^{2}$ & G31 & $a$ & $b$ & $\mathrm{R}^{2}$ \\
\hline $65 / 96$ & $\begin{array}{c}1.003 \\
(13.52)\end{array}$ & $\begin{array}{c}-0.043 \\
(-5.95)\end{array}$ & 0.753 & $65 / 96$ & $\begin{array}{c}0.909 \\
(50.12)\end{array}$ & $\begin{array}{c}-0.030 \\
(-19.51)\end{array}$ & 0.810 \\
\hline $65 / 79$ & $\begin{array}{c}0.976 \\
(75.29)\end{array}$ & $\begin{array}{c}-0.035 \\
(-27.80)\end{array}$ & 0.977 & $65 / 79$ & $\begin{array}{c}0.994 \\
(47.84)\end{array}$ & $\begin{array}{c}-0.036 \\
(-16.88)\end{array}$ & 0.967 \\
\hline $80 / 96$ & $\begin{array}{c}1.081 \\
(14.73)\end{array}$ & $\begin{array}{c}-0.054 \\
(-8.20)\end{array}$ & 0.744 & $80 / 96$ & $\begin{array}{c}0.894 \\
(22.34)\end{array}$ & $\begin{array}{c}-0.031 \\
(-5.03)\end{array}$ & 0.680 \\
\hline
\end{tabular}

On constate que, pour chacun des groupes concernés, et pour chacune des périodes (globale, initiale ou finale), non seulement la corrélation entre

9 Voir tableau précédent où sont reportés les tests sur les deux paramètres $\alpha$ et $\beta$ 
les paramètres $\alpha$ et $\beta$ est très forte, mais elle est. en outre très significativement négative ${ }^{10}$.

Cette relation reflète un comportement invariant. Les paramètres marquent une évolution générale de long terme, à la hausse pour l'ordonnée $\alpha$ et à la baisse pour le coefficient directeur $\beta$ de la droite de régression de $\mathrm{FH}$, mais cette tendance s'accompagne de fluctuations de court terme, dans un sens ou l'autre, des deux paramètres (voir graphique 4).

Un résultat apparaît incontestable : quels que soient leurs comportements respectifs, $\alpha$ et $\beta$ évoluent en sens inverse l'un de l'autre. Cette relation se vérifie pour toutes les périodes et pour tous les groupes. La dynamique d'évolution entre les paramètres suggère que la place accordée au paramètre $\beta$ par FH et par la littérature est surestimée tout à la fois comme indicateur de mobilité et comme courroie de transmission des mécanismes d'ajustement entre épargne et investissement domestiques. Il s'agit donc de s'interroger sur le statut des deux paramètres $\alpha$ et $\beta$ et sur les raisons d'un éventuel pivotement des droites de régression (à court et à long termes) comme sur leur capacité à éclairer l'existence d'un mouvement de mobilité internationale du capital.

Graphique 4: Pivotement de la droite de régression $i / s$
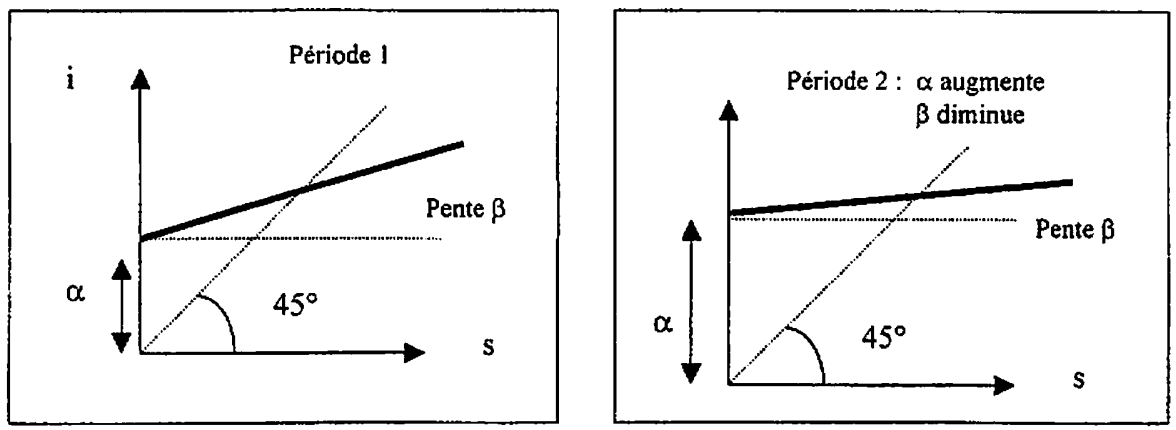

\subsection{Les principes d'analyse}

On a remarqué que pour tous les types de régressions (séries temporelles, coupes transversales ou pooling), la droite de régression $i=\alpha+\beta s$ tendait à se modifier entre la première (1960 ou 1965-79) et la seconde sous-période (1980-96 ou 99) du fait d'une hausse à long terme de $\alpha$ et d'une baisse à long terme de $\beta$.

En outre, l'analyse des divers groupes en coupes instantanées a révélé que ce pivotement de la droite de régression suivait une logique particulière.

10 Ces relations (liés aux régressions sur coupes instantanées) sont similaires à celles qui relient les paramètres $\alpha$ et $\beta$ issus des tests sur coupes periodiques quinquennales. 
Les paramètres sont très fortement liés entre eux et corrélés négativement suivant une relation de la forme de l'équation (9).

Cela donne à penser que la droite de régression pivoterait autour d'un point focal au cours du temps. L'existence de ce « point fixe » peut être justifiée au prix de deux hypothèses qui n'ont rien d'invraisemblable: l'équilibre épargne-investissement pour les ensembles étudiés de pays; l'exogénéité des taux d'épargne.

La première hypothèse se fonde sur la contrainte mondiale d'équilibre entre investissement et épargne : l'économie mondiale dans son ensemble est une économie fermée vérifiant l'égalité de l'épargne et de l'investissement. Or, les quatre groupes retenus représentent chacun une part prépondérante dans l'épargne et l'investissement internationaux ${ }^{1 \text { l }}$.

Le point fixe peut donc être interprété comme le point d'équilibre de la balance courante (impliquant l'égalité entre $i$ et $s$ ). Ce point fixe est défini par la vérification simultanée des deux relations : d'équilibre)

$$
i=\alpha+\beta s \text { et } i=s \text {, ce qui donne (lorsque } i^{*} \text { et } s^{*} \text { sont les taux }
$$

$$
i^{*}=s^{*}=\frac{\alpha}{1-\beta}
$$

L'hypothèse d'exogénéité des taux d'épargne, hypothèse qui fonde la démarche de $\mathrm{FH}$ et que la littérature ne rejette pas, peut être intégrée dans le modèle simple que nous présentons ici. Elle revient à considérer que la mobilité internationale du capital ne peut pas modifier le niveau du taux d'épargne mais uniquement les mouvements de capitaux entre pays et les taux d'investissement de ces pays. Autrement dit, on peut admettre que la mobilité internationale exerce uniquement des effets de type allocatif: elle assure une meilleure répartition de l'épargne mondiale à partir de taux d'épargne prédéterminés sans modifier les comportements d'épargne.

Pour un groupe d'économies interdépendantes, sur une période relativement homogène pour laquelle le point d'équilibre est donné, on doit donc avoir $\alpha /(1-\beta)=s^{*}$, lorsque $s^{*}$ est le taux d'épargne moyen du groupe. Autrement dit, on doit avoir :

$$
\alpha=s^{*}(1-\beta)
$$

Il devient possible de donner une image plus claire des diverses configurations possibles selon les valeurs des paramètres $\alpha$ et $\beta$ dans chaque pays. La relation décrivant le comportement des entrées de capitaux s'écrit sous les hypothèses précédentes:

$$
k=(\beta-1)\left(k-s^{*}\right)
$$

11 Les groupes G15, G7, G22, G31 représentent respectivement $29,65,76$ et $85 \%$ du produit mondial. 
Tableau 5 : Typologie des configurations $\alpha, \beta, k$

\begin{tabular}{|c|c|c|}
\hline & $\begin{array}{c}\beta<1 \text { (ou } \alpha>0) \\
\text { corrélation négative entre } \\
k \text { et } s \text { (cas standard) }\end{array}$ & $\begin{array}{c}\beta>1 \text { (ou } \alpha<0) \\
\text { corrélation positive } \\
\text { entre } k \text { et } s\end{array}$ \\
\hline $\begin{array}{c}s<s^{*} \\
\text { Pays à taux d'épargne } \\
\text { relativement faible }\end{array}$ & Cas I $: k>0$ & Cas II $: k<0$ \\
\hline $\begin{array}{c}s>s^{*} \\
\text { Pays à taux } \\
\text { d'épargne relativement fort }\end{array}$ & Cas III $: k<0$ & Cas IV $: k>0$ \\
\hline
\end{tabular}

Quatre cas de figure (tableau 5) sont alors définis selon le signe de chacun des deux termes de droite.

Les résultats par pays précédemment établis peuvent alors être classés de façon analytique comme il est indiqué dans le tableau 6.

On remarque que les valeurs des paramètres observent le schéma prévu. Les pays ayant un $\beta$ compris entre 0 et 1 se caractérisent par un $\alpha$ positif; les pays ayant un $\beta>1$ se caractérisent par un $\alpha$ négatif. Seule la Thaïlande pour lequel le $\beta$ est supérieur à l'unité a un $\alpha$ non négatif: mais il est vrai que ce paramètre n'est pas significativement différent de 0 .

Tableau 6: Valeurs estimées de $\alpha, \beta$ et $k$ sur la longue période $65-96$

\begin{tabular}{|c|c|c|c|c|c|}
\hline \multicolumn{5}{|c|}{$\beta<1$ (cas standard) } & $\beta>1$ \\
\hline \multicolumn{2}{|c|}{$s^{*}=23.635$} & $\beta<0$ & $\beta=0$ (n s.) & $0<\beta<1$ & $\beta>1$ \\
\hline \multirow{2}{*}{$s<s^{*}$} & \multirow{2}{*}{$\begin{array}{l}\alpha<0 \\
\alpha>0\end{array}$} & & & & ESP, FRA, I'TA, SWE \\
\hline & & & MEX & $\begin{array}{c}\text { ARG, ACS, BEL, BRA, CAN } \\
C H L, \text { GBR, GRC, NZL, } \\
\text { PR.T, USA }\end{array}$ & \\
\hline \multirow{3}{*}{$s>s^{*}$} & \multirow{3}{*}{$\begin{array}{l}\alpha=0 \\
\alpha>0\end{array}$} & & & & $\begin{array}{c}\text { ALT, CHE, DNK, FIN, } \\
J P N, N L D, \text { THA }\end{array}$ \\
\hline & & & & & THA \\
\hline & & IRL & LUX, NOR & DEU, IDN, KOR, SGP, ZAF & \\
\hline
\end{tabular}

Pour les signes attendus de $k$, les observations sont moins favorables: 13 pays sur les 31 (marquées en italique) n'ont pas des mouvement de capitaux du signe attendu. On peut penser que ce résultat est imputable à l'hypothèse implicite forte qui a présidé à la construction du tableau : celle de l'homogénéité de la période. Effectivement, pour 6 des 13 pays concernés, les mouvement de capitaux ont changé de signe entre les deux sous-périodes ${ }^{12}$.

L'instabilité sur le long terme des comportements (valeurs des paramètres $\beta$ et des taux d'épargne) explique donc que le classement des pays

12 II s'agit du Brésil, du Chili, de la Fintande, du Danemark, de la Corée et de Singapour qui sont passés d'une situation de demandeurs de capitaux $(k>0)$ à une situation d'offreurs $(k<0)$. 
Tableau 7 : Estimations des $\beta$ sur séries temporelles, sous-périodes 1965-79 et $1980-96$

\begin{tabular}{|c|c|c|c|c|}
\hline \multicolumn{5}{|c|}{ Classement des pays selon les valeurs de $\beta$ obtenus sur séries temporelles } \\
\hline & $\beta<0$ & $\beta=0$ (non significatif) & $0<\beta<1$ & $\beta>1$ \\
\hline $\begin{array}{r}1965- \\
1979\end{array}$ & & $\begin{array}{c}\text { ESP, GBR, IRL, ITA, LLX } \\
\text { NZI, ZAF }\end{array}$ & $\begin{array}{l}\text { ARG, AUS, AUT, BEL, BRA, CAN, } \\
\text { CHL, DEU, DNK, FRA, IDN, JPN, } \\
\text { KOR, NLD, PRT, SGP, SWE, 'THA }\end{array}$ & $\begin{array}{l}\text { CHE, FIN, GRC, } \\
\text { MEX, NOR, USA }\end{array}$ \\
\hline $\begin{array}{l}1980- \\
1996\end{array}$ & IRL & $\begin{array}{l}\text { DEU, DNK, ESP, GBR, } \\
\text { IDN, MEX, NLD, NOR, } \\
\text { PRT, SGP, SWE }\end{array}$ & $\begin{array}{l}\text { ARG, BEL, BRA, CAN, CHL, GRC, } \\
\text { JPN, KOR., LUX, USA, ZAF }\end{array}$ & $\begin{array}{l}\text { AUS, AUT, CHE, } \\
\text { FIN, FRA, ITA, } \\
\text { NZL, THA }\end{array}$ \\
\hline
\end{tabular}

diffère selon les deux sous-périodes. Le tableau 7 rend compte de la déformation du classement des pays en fonction des valeurs de $\beta$.

Plus généralement, et sur la base de la typologie complète (paramètres $\alpha$ et $\beta$ et taux d'épargne), le classement des pays pour la seconde souspériode fait apparaître des différences notables par rapport à celui établi précédemment pour la longue période 1965/96. (Tableau 8). Les relations prévues entre les paramètres sont conservées (y compris pour le cas particulier de la Thaïlande). Pour le classement des pays selon le signe des mouvements de capitaux, les anomalies deviennent moins nombreuses (elles concernent maintenant 11 pays sur les 31 ). On remarque qu'elles se produisent surtout pour les pays dont le taux d'épargne est faible et qui devraient être importateurs de capitaux. Or, certains de ces pays connaissent des sorties de capitaux. La raison tient probablement au fait que l'échantillon des 31 pays ne représente pas la totalité de l'économie mondiale (mais seulement la plus grande part du produit mondial). Dès lors, un taux d'épargne apparemment « faible » au sein de ce sous-ensemble des pays parmi les plus « riches » excède vraisemblablement le taux mondial moyen : il n'y a ainsi aucune raison pour qu'il ne soit pas assorti d'exportations de capitaux vers le reste du monde.

Tableau 8: Valeurs estimées de $\alpha, \beta$ et $k$ sur la sous-période 80-96

\begin{tabular}{|c|c|c|c|c|c|}
\hline \multicolumn{5}{|c|}{$\beta<1$ (cas standard) } & $\beta>1$ \\
\hline \multicolumn{2}{|l|}{$s^{*}=22.58$} & $\beta<0$ & $\beta=0$ (n s.) & $0<\beta<1$ & $\beta>1$ \\
\hline \multirow{3}{*}{$s<s^{*}=24.82$} & $a<0$ & & & & $A U S$, FRA, I'TA, NZL \\
\hline & $\alpha=0$ & & & & \\
\hline & $\alpha>0$ & & $\begin{array}{l}D E U, D N K, \mathrm{ESP} \\
\mathrm{GBR}, \mathrm{PRT}, S W E\end{array}$ & $\begin{array}{c}A R G, B E L, B R A, C A N \\
\text { CHL, GRC, USA }\end{array}$ & \\
\hline \multirow{3}{*}{$s>s^{*}$} & $\alpha<0$ & & & & $A U T, C H E, F I N$ \\
\hline & $\alpha=0$ & & & ZAF & THA \\
\hline & $\alpha>0$ & IRI & $\begin{array}{c}\text { IDN, MEX, NLD, } \\
\text { NOR, SGP, }\end{array}$ & JPN, KOR, LUX & \\
\hline
\end{tabular}

Dans le cas standard (standard au sens de résultats des régressions en coupes : $0<\beta<1$ et $\alpha>0$ ), les choses sont assez simples et conformes à 
l'intuition. Les pays à taux d'épargne relativement faible sont des importateurs de capitaux alors que les pays dont le taux d'épargne est relativement élevé vont exporter des capitaux.

En revanche, lorsque les valeurs des paramètres sont atypiques, les entrées de capitaux sont d'autant plus importantes que le taux d'épargne domestique est fort: les apports extérieurs de capitaux sont bien corrélées positivement avec l'épargne domestique. En outre, un pays dont le taux d'épargne est initialement élevé et qui exporte des capitaux (entrées de capitaux négatives, ce qui suppose que l'indicateur $\beta$ soit inférieur à l'unité, et ce qui correspond au cas standard de la littérature) pourrait paradoxalement devenir importateur net de capitaux si la valeur de $\beta$ changeait pour devenir supérieure à l'unité ${ }^{13}$.

Une écriture alternative de la relation $k=(\beta-1)\left(s-s^{*}\right)$ en fonction, cette fois, du paramètre oublié de la méthodologie de $\mathrm{FH}$, le coefficient $\alpha$, est encore plus éclairante.

On a en effet: $k=(\beta-1)\left(s-s^{*}\right)=-\alpha s^{*}\left(s-s^{*}\right)$, soit :

$$
k=\alpha\left(1-\frac{s}{s^{*}}\right)
$$

Les entrées nettes de capitaux sont alors déterminées par deux variables:

- La variable $\alpha$ qui mesure le potentiel d'apport de capital autonome (nécessairement positif si on considère le système financier mondial, c'est-à-dire les régressions en coupes);

- Un second terme qui exprime le taux d'utilisation de ce potentiel, terme qui est fonction inverse du taux intérieur d'épargne.

Chaque pays obtient de manière « autonome " (c'est-à-dire indépendamment du comportement d'épargne des agents domestiques) un potentiel $\alpha$ d'entrées nettes de capitaux. Ce potentiel croît avec la globalisation financière, ce qui entraîne une augmentation du potentiel d'entrées autonomes de capitaux. Mais, dans un pays donné, ce potentiel n'est pas systématiquement réalisé.

Les entrées effectives de capitaux dépendent aussi du taux d'utilisation de ce potentiel. Ce taux est fonction des comportements d'épargne intérieure $s$ par rapport à cette sorte de "norme internationale » qui est le taux mondial moyen d'épargne $s^{*}$. On peut considérer que ce second terme fait intervenir un effet de substitution entre épargne extérieure et épargne intérieure.

Plus le taux d'épargne $s$ est fort relativement à la norme $s^{*}$, moins les entrées de capitaux deviennent nécessaires, moins le potentiel est utilisé. Tant que le taux domestique s demeure inférieur à $s^{*}$, la part autonome induite par la globalisation financière conduit à des entrées nettes positives

13 Une configuration dynamique qui ne serait sans doute pas sans rappeler celle des États-Unis au cours des quatre dernières décennies. 
de capitaux, malgré l'existence d'un effet de substitution positif en faveur de l'épargne intérieure. En revanche, quand le taux intérieur s devient relativement grand et dépasse la norme $s^{*}$, le pays devient exportateur net de capitaux.

Il est possible de tester la relation entre les deux paramètres, $\alpha=$ $s^{*}(1-\beta)$, sous la forme :

$$
\alpha_{t}=c+s\left(1-\beta_{t}\right)
$$

Dans l'absolu, on devrait vérifier que $c$ n'est pas significativement différent de 0 . Le coefficient $s$ devrait correspondre au taux moyen d'épargne et d'investissement d'équilibre du groupe concerné. Si le coefficient $s$ est proche des taux moyens et si la constante $c$ est non significative, l'hypothèse du pivotement autour du point fixe est vérifiée.

\subsection{Les résultats}

Les tests ont été conduits pour chacun des groupes étudiés sur la période globale comme sur les deux sous-périodes centrées sur 1980. Le tableau 9 regroupe les résultats. Ont été mentionnés, à titre indicatif, les taux moyens d'investissement et d'épargne (pour chaque groupe et chaque période).

Même si la constante $\mathrm{c}$ n'est pas toujours significativement différente de 0 (mais elle l'est dans 7 régressions sur 12), les valeurs trouvées pour le coefficient s sont compatibles avec les valeurs des taux moyens d'investissement et d'épargne dans un grand nombre de cas. Le groupe G7, l'Europe des 15 (G15), l'échantillon le plus important (G31) valident a priori l'hypothèse du pivot, surtout sur la seconde sous-période. On notera que le groupe G22 pose plus de problèmes, probablement parce que n'y jouent pas les effets d'homogénéité (comme pour G7 et G15), ni les effets de masse et d'agrégation (comme pour le groupe le plus nombreux G31).

Ces résultats sont donc globalement encourageants, surtout dans la mesure où la forme que nous avons retenue pour valider le pivot repose sur une hypothèse de constance des taux moyens : cette hypothèse, rendue nécessaire pour simplifier l'écriture des relations à tester, est beaucoup plus forte et restrictive que celle d'exogénéité des taux d'épargne.

Le schéma qui se dégage des résultats empiriques est celui d'un pivotement des droites de régression (voir graphique 5) autour d'un point fixe qui serait le point d'équilibre de chaque groupe de pays. Sur le long terme, le sens du pivotement est clair et peut être représenté graphiquement : l'ordonnée à l'origine augmente alors que la pente (le coefficient directeur) de la droite décroît.

Le pivotement de la droite de régression se traduit par conséquent :

- Pour les pays dont l'épargne est faible, par la possibilité de mobiliser plus d'épargne externe (entrées de capitaux accrues), donc de mieux satisfaire leurs besoins d'investissement. 
Tableau 9 : Tests du pivotement de la droite de régression

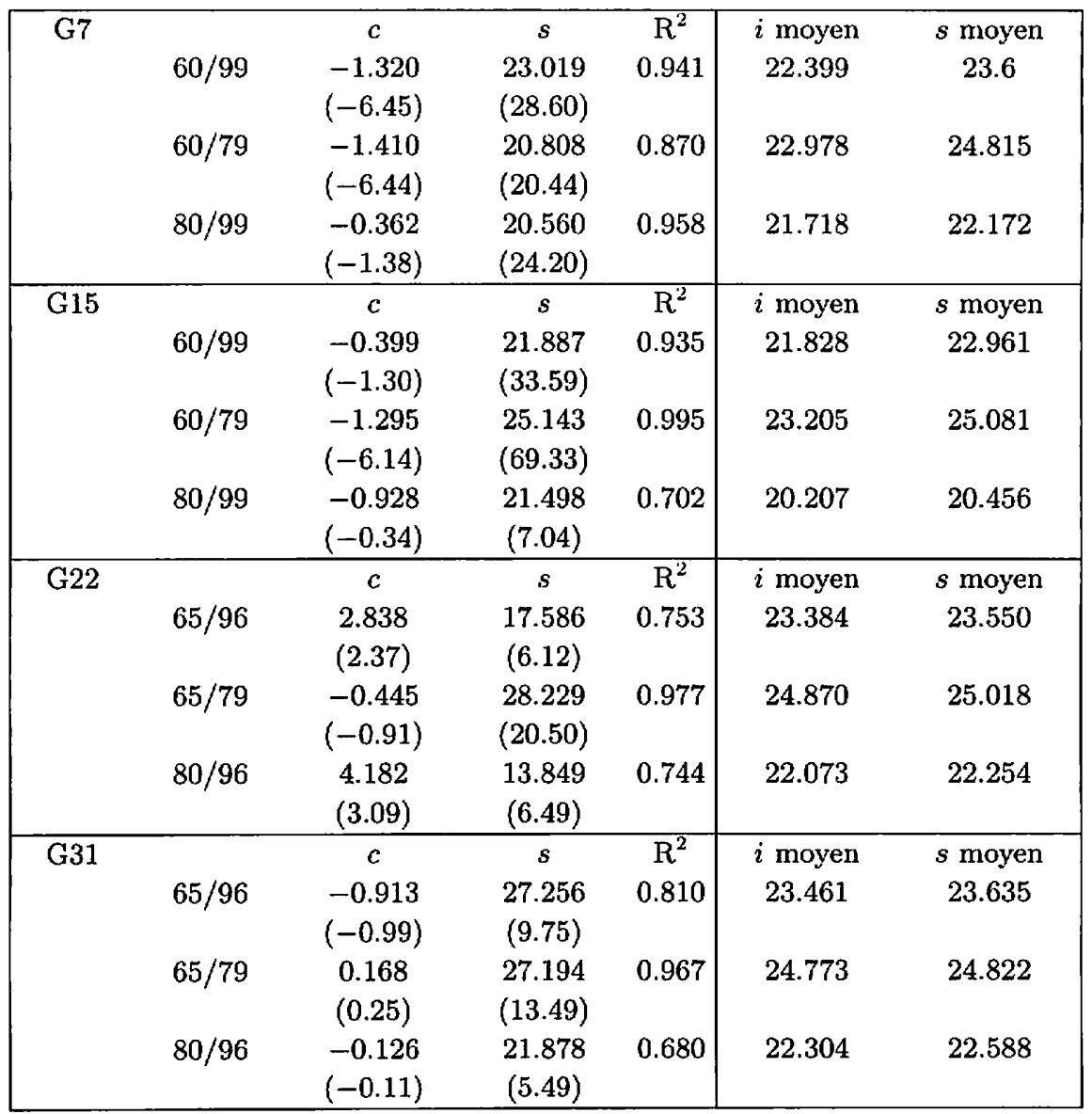

- Pour les pays dont l'épargne est élevée, par la possibilité de trouver plus de débouchés : les sorties de capitaux s'accroissent et les prêteurs trouvent des opportunités d'investissement plus nombreuses.

Évidemment, ces observations mériteraient d'être interprétées à la lumière d'un modèle de macroéconomie internationale explicite, objet qui dépasse le cadre de cet article mais qui constitue indubitablement un prolongement possible de ce dernier.

\section{Conclusion}

La revue de la littérature nous avait conduit à fixer trois axes directeurs pour notre travail économétrique. 
Graphique 5 : Le pivotement de la relation entre $i$ et $s$

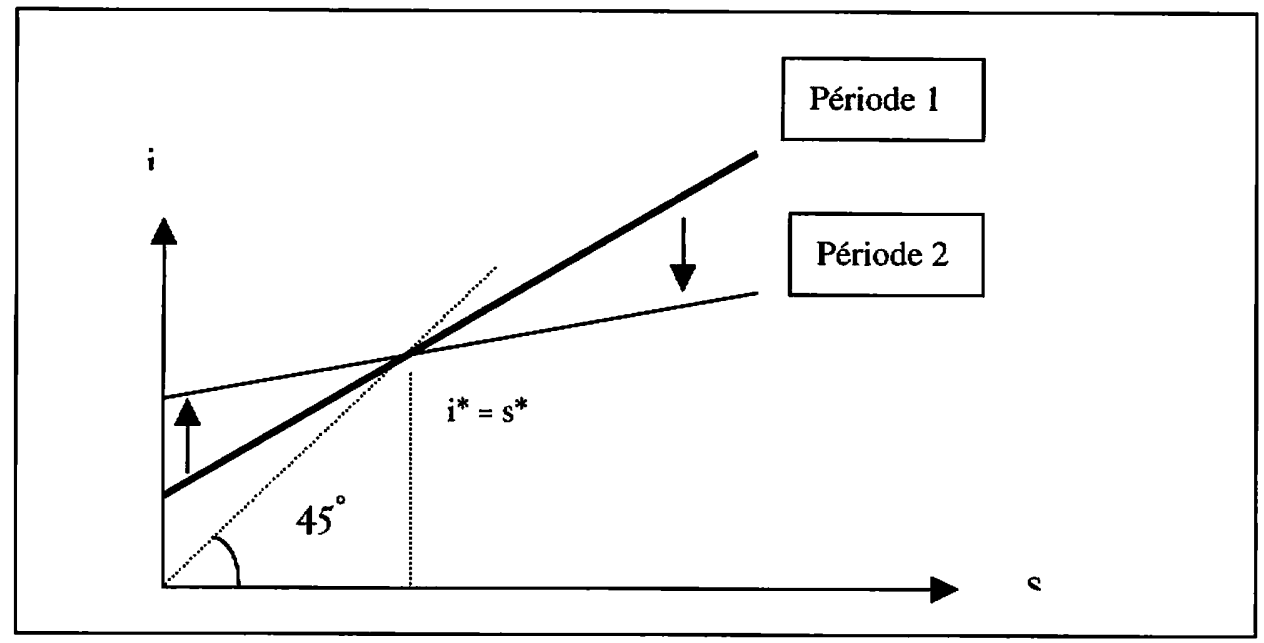

a) Si plusieurs raisons suggéraient qu'un degré élevé de corrélation entre taux d'épargne et taux d'investissement n'était pas contradictoire avec l'existence de marchés financiers interconnectés, cela ne devait pas nous conduire à rejeter la démarche $\mathrm{FH}$. Même si le niveau des paramètres testés était difficile à interpréter, rien ne s'opposait à tirer des enseignements du sens de variation de ces paramètres sur des périodes significatives. C'est pourquoi l'étude économétrique s'est particulièrement intéressé aux changements dans la valeur des paramètres.

b) Face aux deux lignes d'investigation économétrique envisageables (l'approche temporelle par pays; l'approche transversale pour un groupe de pays), plusieurs raisons nous invitaient à privilégier l'approche transversale pour appréhender la mobilité internationale des capitaux car cette dernière concerne par définition le système international pris comme un tout. C'est pourquoi l'étude économétrique a pris soin de bien distinguer les deux classes de phénomènes concernés par l'approche pays et l'approche transversale, de montrer leurs différences et de mettre l'accent sur l'approche en coupes.

c) Nous avions enfin souligné qu'aucune raison n'imposait de se restreindre à l'indicateur $\mathrm{R}^{2}$ ou $\beta$ pour évaluer au plan macroéconomique le sens d'évolution de la mobilité internationale des capitaux. Nous avons signalé qu'il existait des indicateurs complémentaires comme $\alpha$ (ordonnée à l'origine de la droite de régression), c'est-à-dire l'indicateur relatif aux mouvements de capitaux autonomes par rapport à l'épargne intérieure. C'est ainsi que l'étude économétrique a montré toute l'importance qu'il fallait attacher à l'évolution de ce coefficient.

Sur la base de ces trois axes, l'étude empirique a établi un certain nombre d'observations non ambiguës sur le plan économétrique. L'article a 
montré que la logique des changements intervenus dans les flux internationaux de capitaux durant les quarante dernières années pouvait être ramenée à quatre grands faits stylisés :

1) Une augmentation indiscutable de la mobilité internationale des capitaux, augmentation établie à l'aide des tests macroéconomiques habituels à la FH pour les pays industrialisés, grands ou petits. Cette augmentation est repérée par $\beta$ (baisse). L'ampleur de ce changement est considérable et fait contraste avec l'idée généralement rencontrée dans la littérature. Au terme de leur récente recension, Coakley, Kulasi et Smith (1998) écrivent p. 185 : « Néanmoins le puzzle FH n'est en aucun cas résolu et les récentes contributions de Bayoumi et al. (1996) et Sarno et Taylor (1996) invitent à établir l'existence d'un niveau élevé de mobilité du capital dans le cadre de la structure d'analyse $\mathrm{FH}$ ». Les résultats de cet article montrent qu'une grande partie des difficultés se dissout si on affine suffisamment l'étude économétrique.

2) En revanche, les indicateurs usuels font apparaître une relative stabilité de la mobilité internationale des capitaux pour l'ensemble formé par le regroupement des pays industrialisés et des pays émergents, c'est-à-dire l'ensemble concerné par la presque totalité des mouvements internationaux de capitaux. En d'autres termes, la globalisation financière est un phénomène qui se traduit par une divergence avec, d'un côté, le club des pays industrialisés entre lesquels les capitaux deviennent plus mobiles au sens du critère $\mathrm{FH}$, c'est-à-dire un club où les possibilités ouvertes par une plus grande circulation de l'épargne sont effectivement utilisées; et; de l'autre, les pays émergents qui, bien qu'insérés dans les circuits financiers mondiaux, ne semblent pas, au sens du critère $\mathrm{FH}$, profiter au plan macroéconomique des possibilités offertes par une plus grande circulation de l'épargne mondiale. Tant la stationnarité de $\beta$ pour G31 que sa supériorité (par rapport à G22) fait contraste avec l'idée reçue selon laquelle $\beta$ serait plus faible pour les pays en développement.

3) La croissance de la mobilité internationale des capitaux résulte non seulement d'une baisse de $\beta$ mais également d'un accroissement de la « part autonome » des flux nets de capitaux par rapport aux taux d'épargne. On peut considérer qu'il s'est produit un «pivotement » des droites de régression autour d'un point fixe donné par le taux d'épargne mondial. Les analyses précédentes n'ont pas seulement réinterprété la signification du paramètre $\beta$, initialement conçu comme un indicateur de mobilité dans les droites de régression en coupes; ni seulement montré que l'interprétation des changements de ce paramètre était éclairée par la prise en compte des changements de la valeur de $\alpha$; elles ont mis en évidence l'existence d'un point fixe dans les transformations intervenues dans les flux internationaux de capitaux.

4) Le positionnement des pays par rapport au point fixe et selon la valeur prise par $\beta$ (en séries temporelles) permet alors de construire une grille de classification entre différentes configurations, classification qui 
laisse apparaître l'existence de modèles nationaux différenciés au sein du groupe des pays industrialisés ou émergents tant en ce qui concerne les ajustements investissement-épargne qu'en ce qui concerne l'impact macroéconomique de la mobilité des capitaux. Ce résultat fait également contraste avec l'idée reçue selon laquelle tous les pays seraient égaux devant la «globalisation financière » et que celle-ci pourrait être conçue comme un mouvement général doté d'homogénéité. 


\section{Bibliographie}

Artis M. J. et T. A. Bayoumi (1989), "Saving, Investment, Financial Integration, and the Balance of Payments", International Monetary Fund Working Paper, $\mathrm{n}^{\circ}$ 89/102, International Monetary Fund, décembre.

Backus D. K., P. J. Kehoe et F. E. Kykland (1992), "International Real Business Cycles", Journal of Political Economy, 100, p. 584-96.

Baxter M. et M. J. Cruccini (1993), "Explaining Saving-Invesment Correlations", American Economic Review, 36, p. 821-854.

Bayoumi T. A. (1990), "Saving-Investment Correlations", International Monetary Fund Staff Papers, 37, (juin), p. 360-87.

Bayoumi T. A. et A. K. Rose (1993), "Domestic Savings and Intra-National Capital Flows", European Economic review, 37 (août), p. 1197-1202.

Berdot J. P., G. Kébabdjian et J. Léonard (1999), «La dynamique du système monétaire et financier international », 14, 4, Revue Française d'Économie, p. 87-120.

Coakley J., F. Hasan et R. Smith (1999), "Saving, Investment, and Capital Mobility in LDCs", Review of International Economics, 7, 4, p. 632640.

Coakley J., F. Hasan et R. Smith (1998), "The Feldstein-Horioka Puzzle and Capital Mobility: A Review", International Journal of Finance and Economics, 3, 4, p. 169-188.

Coakley J., F., F. Kulasi et R. Smith (1996), "Current account solvency and the Feldstein-Horioka puzzle", The Economic Journal, 106 (mai), p. 620-627.

Dooley M., J. Frankel et D. J. Mathieson (1987), "International capital mobility : what do saving-investment correlations tell us ?", IMF Staff Papers, 34, p. 503-29.

Engel C. et K. Kletzer (1989), "Saving and Investment in an Open Economy with Non-Traded Goods", International Economic Review, 30 (novembre), p. 735-752.

Feldstein M. (1983), "Domestic saving and international capital movements in the long run and the short run", European Economic Review, 21, p. $129-151$.

Feldstein M. et C. Horioka (1980), "Domestic Saving and International Capital Flows", Economic Journal, 90 (juin), p. 314-329.

Feldstein M; et P. Bacchetta (1991), "National Saving and International Investment", in B. D. Bernheim et J. B Shoven (eds), National Saving and Economic performance, University of Chicago Press, p. 201-220.

Fieleke N. (1982), "National saving and international investment", in Federal Reserve Bank of Boston, Saving and Government Policy.

Finn M.G., (1990), « On savings and investment dynamics in a small open economy », Journal of International Economics, 29, p. 1-21. 
Frankel J. A. (1986), "International Capital Mobility and Crowding-Out in the US Economy: Imperfect Integration of Financial Markets or of Goods Markets" in R. W. Hafer (ed), How Open is the US Economy?, Lexington, Mass., and Toronto Heath, p. 33-67.

Frankel J. A. (1993), "Quantifying International Capital Mobility in the 1980s", in J. A. Frankel (ed), On exchange Rates, MIT Press, p. 4169.

Ghosh A. R. (1995), "International capital mobility amongst the major industrialed countries : too little or too much", The Economic Journal, 105 (janvier), p. 107-128.

Grinols E. L. (1996), "The link between Domestic Investment and Domestic Savings in Open Economies : Evidence from Balanced Stochastic Growth", Review of International Economics, 4, 2, 119-140.

Harberger A. (1980), "Vignettes on the World Capital Market", American Economic Review, 70, 331-37.

Higgins M. (1998), "Demography, national savings, and international capital flows", International Economic Review, 39, 2, p. 343-369.

Moosa I. A. (1997), "Resolving the Feldstein-Horioka puzzle", Economia Internazionale, L 3, août, p.437-457.

Murphy R. (1984), "Capital mobility and the relationship between saving and investment in OECD countries", Journal of International Money and Finance, 3, p.327-342.

Murphy R. (1984), "Capital mobility in the world economy:theory and measurement", Carnegie Rochester Conference Series, 24, p. 55-104.

Murphy R. (1986), "Productivity Shocks, Non-Traded Goods and Optimal Accumulation", European Economic Review, 30, p. 1081-1095.

Obstfeldt M. (1986), "Capital Mobility in the World Economy: Theory and measurement", Carnegie-Rochester Conference Series on Public policy, 24 (été), p. 55-103.

Obstfeldt M. (1995), "International Capital Mobility in the 1990s" in P. B. Kenen (ed), Understanding Interdependence, The macroeconomics of the open economy, Princeton University Press.

Obstfeldt M. et K. Rogoff (1996), Foundations of International Macroeconomics, The MIT Press.

Sachs J. D. (1981), "The Current Account and Macroeconomic Activity in the 1970s"', Brookings Papers in Economic Activity, 12, p. 1089-92

Sachs J. D. (1982), "The Current Account and Macroeconomic Adjustment Process", Scandinavian Journal of Economics, 84, p. 147-159.

Shibata A. et M. Shintani (1998), "Capital mobility in the world economy: an alternative test", Journal of International Money and Finance, 17, p. 741-756. 
Summers L. (1988), "Tax policy and international competitiveness", in J. Frankel (ed.), International Aspects of Fiscal Policies, University of Chicago Press, p. 349-75.

Taylor A. M. (1994), "Domestic saving and international capital flows reconsidered", NBER Working Papers n 4892 (oct.).

Tesar, L. (1991), "Savings, Investment, and International Capital Flows", Journal of International Economics, 31, p. 55-78.

Tobin J. (1983), "Comments. 'Domestic saving and international capital movements in the long run and the short run' by Feldstein", European Economic Review, 21, p. 153-156.

Turnovsky S. J. (1997), International Macroeconomics Dynamics, The MIT Press.

Wesphal U. (1983), "Comment on 'Domestic Saving and International capital Movements in the Long Run and the Short Run"', European Economic Review, 21, p. 157-59.

Wong D. Y. (1990), "What do saving-investment relationships tell us about capital mobility?", Journal of International Money and Finance, 9, p.60-74. 